This is the post peer-review accepted manuscript of:

E. Idà, T. Bruckmann and M. Carricato, "Rest-to-Rest Trajectory Planning for Underactuated Cable-Driven Parallel Robots," in IEEE Transactions on Robotics, vol. 35, no. 6, pp. 1338-1351, Dec. 2019.

doi: $10.1109 /$ TRO.2019.2931483

The published version is available online at:

http://ieeexplore.ieee.org/stamp/stamp.jsp?tp=\&arnumber=8815936\&isnumber=8922823

(C) 2019 IEEE. Personal use of this material is permitted. Permission from IEEE must be obtained for all other uses, in any current or future media, including reprinting/republishing this material for advertising or promotional purposes, creating new collective works, for resale or redistribution to servers or lists, or reuse of any copyrighted component of this work in other works. 


\title{
Rest-to-Rest Trajectory Planning for Underactuated Cable-Driven Parallel Robots
}

\author{
Edoardo Idà, Tobias Bruckmann and Marco Carricato, IEEE Senior Member
}

\begin{abstract}
This paper studies the trajectory planning for underactuated cable-driven parallel robots (CDPRs) in the case of rest-to-rest motions, when the motion time and the path geometry are prescribed. For underactuated manipulators, it is possible to prescribe a control law only for a subset of the generalized coordinates of the system. However, if an arbitrary motion is prescribed for a suitable subset of these coordinates, the constraint deficiency on the end-effector motions leads to the impossibility of bringing the system at rest in a prescribed time. In addition, the behavior of the system may not be stable, that is, unbounded oscillatory motion of the end-effector may arise. In this paper, we propose a novel trajectory-planning technique that allows the end-effector to track a constrained geometric path in a specified time, and allows it to transition between stable static poses. The design of such a motion is based on the solution of a Boundary Value Problem, formulated as the problem of finding a solution to the differential equations of motion, with constraints on position and velocity at start and end times. To prove the effectiveness of such a method, the trajectory planning of a 6-Degree-of-Freedom spatial $C D P R$ suspended by 3 cables is investigated. Trajectories of a reference point on the moving platform are designed so as to ensure that the assigned path is tracked accurately and the system is brought to a static condition in a prescribed time. Experimental validation is presented and discussed.
\end{abstract}

Index Terms-Cable-driven parallel robots, Underconstrained robots, Underactuated robots, Trajectory planning

\section{INTRODUCTION}

C ABLE-DRIVEN parallel robots (CDPRs) employ cables in place of rigid-body links in order to control the end-effector $(e-e)$ pose. Cables are attached to the endeffector in a parallel topology and are coiled on servocontrolled winches. A CDPR is underactuated if the number of actuators employed for the control of the $e$ - $e$ is less then the number of its degrees of freedom (DoFs). This means that only a sub-set of the generalized coordinates of the $e-e$ can be directly controlled, while the others are determined by the system dynamics. An underactuated $C D P R$ is always underconstrained, namely the dimension of the constraint space spanned by passive and active constraints acting on the $e-e$ is smaller than six [2], so that the $e$ - $e$ preserves some degrees of freedom once actuators are locked. In some cases, a completely actuated $C D P R$ may be underconstrained, because either some cables become

E. Idà and M. Carricato are with the Dept. of Industrial Engineering, University of Bologna, 40137 Bologna, Italy (e-mails: edoardo.ida2@unibo.it, marco.carricato@unibo.it).

T. Bruckmann is with the Chair for Mechatronics, University of DuisburgEssen, 47057 Duisburg, Germany (e-mail: tobias.bruckmann@uni-due.de).

A preliminary version of this paper was presented at the 3rd Int. Conf. on Cable-Driven Parallel Robots, 2017, Québec City, Canada 1 slack during operation [3] or active cables are mounted on movable shuttles [4], [5].

The use of underactuated $C D P R s$, equipped with a limited number of cables, is justified in several applications, in which the task to be performed requires a limited number of controlled freedoms or a limitation of mobility is acceptable in order to enhance accessibility or decrease complexity. While a rich literature is available describing fully and overconstrained CDPRs, concerning kinematics [6], [7], workspace [8]-[13], dynamics [14]-[19], cable modelling [20] and interference [21], little research has been conducted on underactuated ones, mainly focusing on specific applications [22], [23] or kinematics [3], [24], [25].

A major challenge in the analysis of underactuated systems is the trajectory planning for point-to-point motions. This problem has been addressed in the past especially in the case of completely actuated CDPRs. Most notably, in [14- -19 the dynamics of completely actuated CDPRs was exploited in order to compute dynamically feasible trajectories, extending outside their static workspace. When the $C D P R$ is underconstrained but fully actuated (as in [4], [5]), the system is flat [26, and the trajectory planning problem is completely algebraic [5]. Instead, for a generic underactuated (and thus inherently underconstrained) $C D P R$, different techniques must be employed. These characteristics lead to the impossibility of bringing the platform to rest once the transition from the starting point to the ending point is completed, and possibly to the generation of unbounded oscillatory motion of the end-effector.

In 27, a pendulum-like robot was proposed, consisting of a point mass suspended by a single cable. In order for this system to move outside a straight vertical line and perform point-to-point motions, non zero initial condition must be provided. A planar 3-DoF CDPR suspended by 2 cables was proposed in [28]: the authors were able to generate point-to-point $e$-e movements outside the static workspace, by exploiting harmonic motion laws for the cable lengths; however, they were not able to impose constraints on the path to follow or on the transition time. An input-shaping filtering technique was then proposed in 29] for a planar $C D P R$ and in 30 for a spatial model. Generic trajectories were proposed for which the platform oscillations were notably reduced, but not eliminated, even in a simulation environment, mainly due to the approximation of the robot natural frequency used in the input shaper. Moreover, the nature of the input shaping filter does not allow precise tracking of geometrical paths to be achieved, since the nominal path is modified by the filter. Our objective is to develop a trajectory-planning method suitable for a 


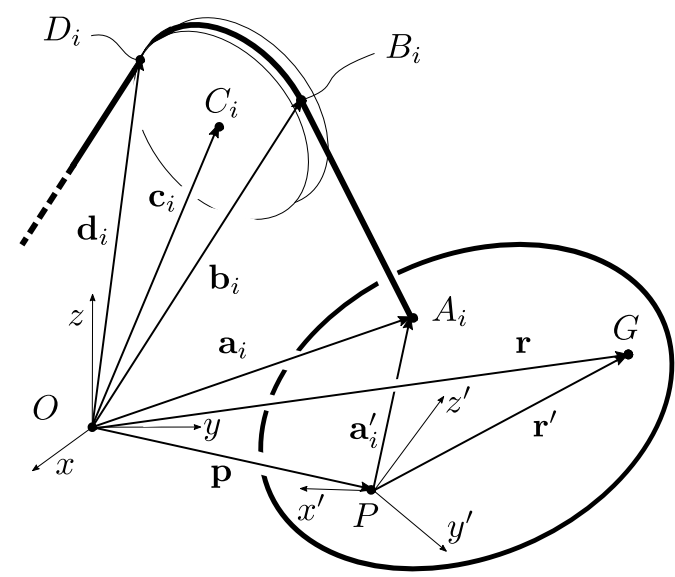

Figure 1. Geometry of the cable-driven robot

stationary setpoint transition, when both the transition path for a suitable subset of the generalized coordinates of the $e$ $e$ and the transition time are assigned. A stationary setpoint transition refers to a point-to-point motion of the platform that is performed between two static equilibrium poses. In the preliminary conference version of this paper [1], we proposed a novel technique to specify a constrained path for a subset of the generalized coordinates of a 3-DoF planar system; this path can be tracked in a specified time and allows the platform to transition between static poses. The novel contributions of this enhanced version are: (i) the extension of the method to a generic underactuated 6-DoF spatial $C D P R$, whose dynamic model is more complex, due to the presence of a larger number of uncontrollable $e-e$ motions and the presence of swivel pulleys, which have a significant influence on the manipulator dynamics; (ii) the introduction of additional details regarding the formulation and the solution of the planning problem in Sections IV-B and $\mathrm{IV}-\mathrm{C}$ (iii) the experimental validation of the method on a $6-D o F$ spatial prototype.

The outline of the paper is as follows. Sections II and III present, respectively, the extended kinematic model of the cable transmission, accounting for swivel-pulley kinematics, and the dynamic model of the mobile platform. Section IV proposes a generic trajectory-planning method that is based on the solution of the BVP arising from the platform dynamics. In Section $\mathrm{V}$ the implementation of such a method is proposed in the case of a spatial robot, and the results of experimental tests on a prototype are presented and discussed. Finally, in Section $\mathrm{VI}$. conclusions are drawn.

\section{Kinematic Model}

\section{A. Finite Kinematics}

A $C D P R$ consists of a mobile platform coupled to the base by $n$ cables, which can be coiled and uncoiled by motorized winches. If $n$ is less than the dimension of the motion space $h$ ( $h=3$ for planar motions and $h=6$ for spatial motions), then the $C D P R$ is underactuated. In the following, $O x y z$ is an inertial frame, whereas $P x^{\prime} y^{\prime} z^{\prime}$ is a mobile frame attached to the moving platform, whose pose

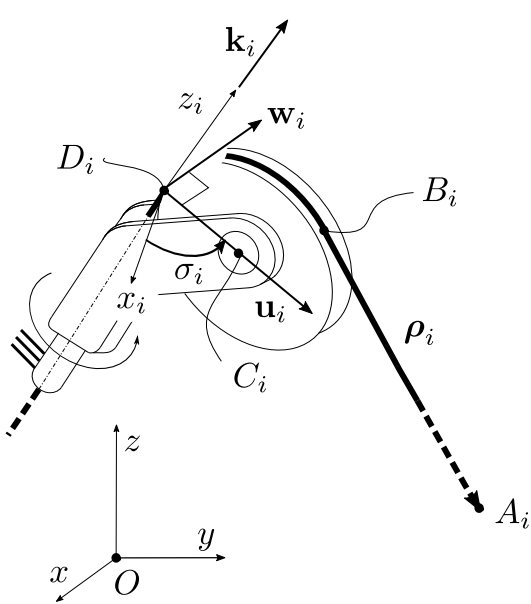

Figure 2. Geometry of the swivel pulley

is described by the position vector $\mathbf{p}$ of $P$, and the rotation matrix $\mathbf{R}$ (Fig. 11. $\mathbf{R}$ is parametrized by the Euler angles $\boldsymbol{\epsilon}=[\phi, \theta, \chi]^{T}$ according to the $x y z$ convention, namely:

$$
\mathbf{R}(\phi, \theta, \chi)=\mathbf{R}_{x}(\phi) \mathbf{R}_{y}(\theta) \mathbf{R}_{z}(\chi)
$$

where $\mathbf{R}_{x}, \mathbf{R}_{y}$ and $\mathbf{R}_{z}$ are elementary rotation matrices about $x, y$ and $z$ axes. The platform generalized coordinates are $\mathbf{q}=[\mathbf{p}, \boldsymbol{\epsilon}]^{T}$.

The $i$-th cable, modelled as massless and inextensible, is guided into the workspace by a swivel pulley [7], [31], and it is attached to the platform at point $A_{i}$ (Figs. 1 2 2] $G$ is the platform center of mass. If the coordinates of $G$ and $A_{i}$ in the mobile frame are described by vectors ${ }^{P} \mathbf{r}^{\prime}$ and ${ }^{P} \mathbf{a}_{i}^{\prime}$, their coordinates in the inertial frame are:

$$
\begin{aligned}
\mathbf{r} & =\mathbf{p}+\mathbf{r}^{\prime}=\mathbf{p}+\mathbf{R}^{P} \mathbf{r}^{\prime} \\
\mathbf{a}_{i} & =\mathbf{p}+\mathbf{a}_{i}^{\prime}=\mathbf{p}+\mathbf{R}^{P} \mathbf{a}_{i}^{\prime}
\end{aligned}
$$

The $i$-th swivel pulley has center $C_{i}$, radius $r_{i}$, and is mounted on a hinged support, whose swivel axis $z_{i}$ is tangent to the pulley (Fig. 2). The fixed point $D_{i}$ where the $i$-th cable enters the pulley's groove is on the $z_{i}$-axis. Its position vector in $O x y z$ is $\mathbf{d}_{i}$. It is convenient to define an additional fixed reference frame $D_{i} x_{i} y_{i} z_{i}$ attached to $D_{i}$, whose orientation in $O x y z$ is described by the (constant) rotation matrix $\mathbf{R}_{i}=\left[\begin{array}{lll}\mathbf{x}_{i} & \mathbf{y}_{i} & \mathbf{z}_{i}\end{array}\right]$. The $i$-th cable exit point from the pulley groove is denoted by $B_{i}$, whereas the vector $A_{i}-B_{i}$, which is tangent to the pulley, is $\boldsymbol{\rho}_{i}$. Pulley kinematic modelling has a significative impact in both the kinematics and the dynamics of the CDPR platform, since it accounts both for the varying amount of cable that is wrapped onto the pulley and for the variation of the cable exit point positions. Although pulley kinematics may have a limited impact on the finite kinematics of the experimental setup

\footnotetext{
${ }^{1}$ In the prototype used for the experimentation discussed in Section $\mathrm{V}$, each cable is attached to the platform by means of a custom-made lightweight universal joint centered in $A_{i}$, which ensures that the cable axis precisely passes through a fixed point on the platform. Moreover, winches are grooved so that cable spiralling is eliminated and the transmission ratio between the motor angle and the cable length is approximately constant.
} 
discussed in Section it has a relevant influence on its differential kinematics (the magnitude of the acceleration of point $P$ on the $e$ - $e$ may vary up to $50 \%$ if no pulley transmission is considered). Furthermore, the variation of the cable exit points modifies the direction of the cable tensions that are acting on the platform. The kinematics of swivel pulleys was studied in [7], [31] and additional references can be found in 32 . The implementation included in our model is presented in the following.

If friction in the pulley hinge is negligible, the line through $A_{i}$ and $B_{i}$ and the $z_{i}$-axis must be coplanar, due to the rotational equilibrium of the pulley around $z_{i}$. As a consequence, the unit vector $\mathbf{k}_{i}$ along $z_{i}$ and vector $\boldsymbol{\rho}_{i}$ completely define the pulley plane. If $\sigma_{i}$ is the angle between the coordinate plane $x_{i} z_{i}$ and the pulley plane (Fig. 2), the unit vector $\mathbf{w}_{i}$ perpendicular to the latter is:

$$
\mathbf{w}_{i}=-\sin \sigma_{i} \mathbf{i}_{i}+\cos \sigma_{i} \mathbf{j}_{i}=\frac{\mathbf{k}_{i} \times\left(\mathbf{a}_{i}-\mathbf{d}_{i}\right)}{\left\|\mathbf{k}_{i} \times\left(\mathbf{a}_{i}-\mathbf{d}_{i}\right)\right\|}
$$

where $\mathbf{i}_{i}, \mathbf{j}_{i}$ and $\mathbf{k}_{i}$ are unit vectors along axes $x_{i}, y_{i}$ and $z_{i}$. It can be easily shown that:

$$
\sigma_{i}=\operatorname{atan} 2\left[\left(\mathbf{a}_{i}-\mathbf{d}_{i}\right) \cdot \mathbf{j}_{i},\left(\mathbf{a}_{i}-\mathbf{d}_{i}\right) \cdot \mathbf{i}_{i}\right]
$$

In addition, the unit vector $\mathbf{u}_{i}$ from $D_{i}$ to $C_{i}$ (perpendicular to both $\mathbf{k}_{i}$ and $\mathbf{w}_{i}$ ) is (Fig. 2):

$$
\mathbf{u}_{i}=\frac{\mathbf{c}_{i}-\mathbf{d}_{i}}{\left\|\mathbf{c}_{i}-\mathbf{d}_{i}\right\|}=\cos \sigma_{i} \mathbf{i}_{i}+\sin \sigma_{i} \mathbf{j}_{i}
$$

If $\psi_{i} \in(-\pi, \pi)$ is the angle between $\mathbf{u}_{i}$ and $\mathbf{b}_{i}-\mathbf{c}_{i}$ (Fig. 3), then:

$$
\mathbf{b}_{i}-\mathbf{c}_{i}=r_{i}\left(\cos \psi_{i} \mathbf{u}_{i}+\sin \psi_{i} \mathbf{k}_{i}\right)
$$

and also, due to the closure of polygon $O A_{i} B_{i} C_{i} D_{i}$ :

$$
r_{i}\left(\cos \psi_{i} \mathbf{u}_{i}+\sin \psi_{i} \mathbf{k}_{i}\right)=\mathbf{a}_{i}-\boldsymbol{\rho}_{i}-r_{i} \mathbf{u}_{i}-\mathbf{d}_{i}
$$

By taking the dot product of both sides of Eq. 83 with the unit vector $\left(\cos \psi_{i} \mathbf{u}_{i}+\sin \psi_{i} \mathbf{k}_{i}\right)$, and imposing the perpendicularity condition between $\boldsymbol{\rho}_{i}$ and $\mathbf{b}_{i}-\mathbf{c}_{i}$, we obtain:

$$
r_{i}=\left(\mathbf{a}_{i}-r_{i} \mathbf{u}_{i}-\mathbf{d}_{i}\right) \cdot\left(\cos \psi_{i} \mathbf{u}_{i}+\sin \psi_{i} \mathbf{k}_{i}\right)
$$

and hence:

$$
\cos \psi_{i}\left[\left(\mathbf{a}_{i}-\mathbf{d}_{i}\right) \cdot \mathbf{u}_{i}-r_{i}\right]+\sin \psi_{i}\left(\mathbf{a}_{i}-\mathbf{d}_{i}\right) \cdot \mathbf{k}_{i}-r_{i}=0
$$

By expressing $\sin \psi_{i}$ and $\cos \psi_{i}$ as functions of $t_{i}=\tan \left(\psi_{i} / 2\right)$, Eq. 10 can be written as:

$$
\left(1-t_{i}^{2}\right)\left[\left(\mathbf{a}_{i}-\mathbf{d}_{i}\right) \cdot \mathbf{u}_{i}-r_{i}\right]+2 t_{i}\left(\mathbf{a}_{i}-\mathbf{d}_{i}\right) \cdot \mathbf{k}_{i}-\left(1+t_{i}^{2}\right) r_{i}=0
$$

which yields:

$$
t_{i}^{2}-2 \frac{\left(\mathbf{a}_{i}-\mathbf{d}_{i}\right) \cdot \mathbf{k}_{i}}{\left(\mathbf{a}_{i}-\mathbf{d}_{i}\right) \cdot \mathbf{u}_{i}} t_{i}+\frac{2 r_{i}}{\left(\mathbf{a}_{i}-\mathbf{d}_{i}\right) \cdot \mathbf{u}_{i}}-1=0
$$

Equation (12) provides two angles, which define the two points of intersection of the two tangent lines to the pulley passing through $A_{i}$. In our case, we are only interested in

\footnotetext{
${ }^{2}$ It is worth pointing out that any industrial implementation of cable robots will likely use larger pulley radii than the ones adopted in our implementation, since the ratio of pulley radius to cable radius must be as large as possible in order to guarantee an acceptable lifetime of the cable, thus increasing the effects of pulley kinematics.
}

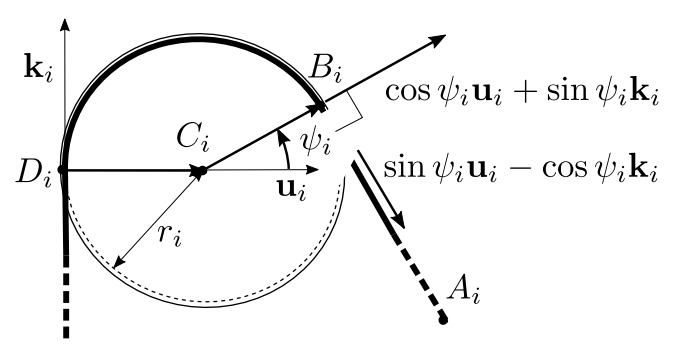

Figure 3. Front view of the Swivel Pulley

the greater angle, because the cable is wrapped clockwise on the pulley from the frame to the platform, namely:

$$
\begin{aligned}
\psi_{i}=2 \operatorname{atan}\left[\frac{\left(\mathbf{a}_{i}-\mathbf{d}_{i}\right) \cdot \mathbf{k}_{i}}{\left(\mathbf{a}_{i}-\mathbf{d}_{i}\right) \cdot \mathbf{u}_{i}}+\right. \\
\left.+\sqrt{1-\frac{2 r_{i}}{\left(\mathbf{a}_{i}-\mathbf{d}_{i}\right) \cdot \mathbf{u}_{i}}+\left(\frac{\left(\mathbf{a}_{i}-\mathbf{d}_{i}\right) \cdot \mathbf{k}_{i}}{\left(\mathbf{a}_{i}-\mathbf{d}_{i}\right) \cdot \mathbf{u}_{i}}\right)^{2}}\right]
\end{aligned}
$$

Finally, vector $\boldsymbol{\rho}_{i}$ can be computed from Eq. 8] as:

$$
\boldsymbol{\rho}_{i}=\mathbf{a}_{i}-\mathbf{d}_{i}-r_{i}\left[\left(1+\cos \psi_{i}\right) \mathbf{u}_{i}+\sin \psi_{i} \mathbf{k}_{i}\right]
$$

and thus (Fig. 3):

$$
\begin{aligned}
\left\|\boldsymbol{\rho}_{i}\right\| & =\boldsymbol{\rho}_{i} \cdot\left(\sin \psi_{i} \mathbf{u}_{i}-\cos \psi_{i} \mathbf{k}_{i}\right)= \\
& =\left(\mathbf{a}_{i}-\mathbf{d}_{i}\right) \cdot\left(\sin \psi_{i} \mathbf{u}_{i}-\cos \psi_{i} \mathbf{k}_{i}\right)-r_{i} \sin \psi_{i}
\end{aligned}
$$

In the end, the geometrical constraint that the $i$-th cable imposes on the platform is:

$$
\boldsymbol{\rho}_{i}^{T} \boldsymbol{\rho}_{i}-\left[l_{i}-r_{i}\left(\pi-\psi_{i}\right)\right]^{2}=0
$$

where $l_{i}$ is the $i$-th total cable length, comprising the rectilinear cable length $\left\|\boldsymbol{\rho}_{i}\right\|$ and the wrapped cable length $\widehat{B_{i} D_{i}}$.

\section{B. Infinitesimal Kinematics}

The velocity and acceleration of $G$ and $A_{i}$ are:

$$
\begin{aligned}
\dot{\mathbf{r}} & =\dot{\mathbf{p}}+\omega \times \mathbf{r}^{\prime} \\
\dot{\mathbf{a}}_{i} & =\dot{\mathbf{p}}+\boldsymbol{\omega} \times \mathbf{a}_{i}^{\prime} \\
\ddot{\mathbf{r}} & =\ddot{\mathbf{p}}+\boldsymbol{\alpha} \times \mathbf{r}^{\prime}+\boldsymbol{\omega} \times\left(\boldsymbol{\omega} \times \mathbf{r}^{\prime}\right) \\
\ddot{\mathbf{a}}_{i} & =\ddot{\mathbf{p}}+\boldsymbol{\alpha} \times \mathbf{a}_{i}^{\prime}+\boldsymbol{\omega} \times\left(\boldsymbol{\omega} \times \mathbf{a}_{i}^{\prime}\right)
\end{aligned}
$$

where $\boldsymbol{\omega}$ and $\boldsymbol{\alpha}$ are the angular velocity and acceleration of the platform, respectively. The angular velocity is linearly dependent on the Euler angles derivatives $\dot{\boldsymbol{\epsilon}}$, namely:

$$
\boldsymbol{\omega}=\mathbf{H}(\boldsymbol{\epsilon}) \dot{\boldsymbol{\epsilon}}=\left[\begin{array}{ccc}
1 & 0 & s_{\theta} \\
0 & c_{\phi} & -s_{\phi} c_{\theta} \\
0 & s_{\phi} & c_{\phi} c_{\theta}
\end{array}\right] \dot{\boldsymbol{\epsilon}}
$$

where $c_{x}=\cos x$ and $s_{x}=\sin x$. Accordingly, the angular acceleration can be calculated as:

$$
\boldsymbol{\alpha}=\mathbf{H} \ddot{\boldsymbol{\epsilon}}+\dot{\mathbf{H}} \dot{\boldsymbol{\epsilon}}
$$

The rate of change of the pulley-plane orientation can be computed from Eqs. (4), (5) and [6. Indeed, since:

$$
\dot{\mathbf{w}}_{i}=-\dot{\sigma}_{i} \mathbf{u}_{i}, \quad \dot{\mathbf{u}}_{i}=\dot{\sigma}_{i} \mathbf{w}_{i}
$$


taking the dot-product of $\mathbf{w}_{i}$ with $\mathbf{a}_{i}-\mathbf{d}_{i}$ and differentiating with respect to time yields, after some rearrangement:

$$
\dot{\sigma}_{i}=\frac{\mathbf{w}_{i} \cdot \dot{\mathbf{a}}_{i}}{\mathbf{u}_{i} \cdot\left(\mathbf{a}_{i}-\mathbf{d}_{i}\right)}
$$

The rate of change of the cable orientation $\psi_{i}$ in the pulley plane can be obtained by differentiating Eq. 110) with respect to time. Taking advantage of Eqs. (15) and [23), $\dot{\psi}_{i}$ can thus be expressed as:

$$
\dot{\psi}_{i}=\frac{\left(\cos \psi_{i} \mathbf{u}_{i}+\sin \psi_{i} \mathbf{k}_{i}\right) \cdot \dot{\mathbf{a}}_{i}}{\left\|\boldsymbol{\rho}_{i}\right\|}
$$

The rate of change of $\boldsymbol{\rho}_{i}$ can be obtained by differentiating Eq. [14 w.r.t. time, namely:

$$
\dot{\boldsymbol{\rho}}_{i}=\dot{\mathbf{a}}_{i}-r_{i}\left[\dot{\sigma}_{i}\left(1+\cos \psi_{i}\right) \mathbf{w}_{i}-\dot{\psi}_{i}\left(\sin \psi_{i} \mathbf{u}_{i}-\cos \psi_{i} \mathbf{k}_{i}\right)\right]
$$

where the velocity $\dot{\mathbf{a}}_{i}$ is in Eq. (18).

Finally, differentiating Eq. 16] w.r.t. time yields:

$$
2 \boldsymbol{\rho}_{i}^{T} \dot{\boldsymbol{\rho}}_{i}-2\left[l_{i}-r_{i}\left(\pi-\psi_{i}\right)\right]\left(\dot{l}_{i}+r_{i} \dot{\psi}_{i}\right)=0
$$

Substituting Eqs. 26 and 21) in Eq. 27) provides the relationship between the cable system kinematics and the end-effector twist $\mathbf{v}=\left[\dot{\mathbf{p}}^{T} \boldsymbol{\omega}^{T}\right]^{T}$, or the generalized velocity $\dot{\mathbf{q}}=\left[\dot{\mathbf{p}}^{T} \dot{\boldsymbol{\epsilon}}^{T}\right]^{T}$. It can be shown by computation that:

$$
\mathbf{J}_{v} \mathbf{v}=\mathbf{J}_{q} \dot{\mathbf{q}}=\mathbf{J}_{l} \dot{\mathbf{l}}
$$

where the $i$-th rows of matrices $\mathbf{J}_{v}, \mathbf{J}_{q}$ and $\mathbf{J}_{l}$ are:

$$
\begin{aligned}
& \mathbf{J}_{v, i}=\left[\begin{array}{ll}
\boldsymbol{\rho}_{i}^{T} & -\boldsymbol{\rho}_{i}^{T} \tilde{\mathbf{a}}_{i}^{\prime}
\end{array}\right] \\
& \mathbf{J}_{q, i}=\left[\begin{array}{ll}
\boldsymbol{\rho}_{i}^{T} & -\boldsymbol{\rho}_{i}^{T} \tilde{\mathbf{a}}_{i}^{\prime} \mathbf{H}
\end{array}\right] \\
& \mathbf{J}_{l, i}=\left[\begin{array}{lllllll}
0_{1} & \cdots & 0_{i-i} & l_{i}-r_{i}\left(\pi-\psi_{i}\right) & 0_{i+1} & \cdots & 0_{n}
\end{array}\right]
\end{aligned}
$$

$\mathbf{i}=\left[\dot{l}_{1}, \ldots, \dot{l}_{n}\right]^{T}$ is a $n$-dimensional array containing the time derivative of cable lengths, and $\tilde{\mathbf{a}}_{i}^{\prime}$ is the skew-symmetric representation of the vector product, namely $\mathbf{a}_{i}^{\prime} \times=\tilde{\mathbf{a}}_{i}^{\prime}$.

\section{DYNAMIC MODEL}

The dynamic model of the $C D P R$ emerges from the differential equation of the platform dynamics, subject to the constraints imposed by cables.

If $\mathbf{f}_{G}$ and $\mathbf{m}_{G}$ are the resultant external force and moment about $G$, the force and moment equilibria about the reference point $P$ yield:

$$
\begin{array}{r}
m \ddot{\mathbf{r}}-\mathbf{f}_{G}-\mathbf{f}_{C}=\mathbf{0} \\
\mathbf{I}_{G} \boldsymbol{\alpha}+\boldsymbol{\omega} \times\left(\mathbf{I}_{G} \boldsymbol{\omega}\right)+m \mathbf{r}^{\prime} \times \ddot{\mathbf{r}}-\left(\mathbf{m}_{G}+\mathbf{r}^{\prime} \times \mathbf{f}_{G}\right)-\mathbf{m}_{C}=\mathbf{0}
\end{array}
$$

where $m$ is the platform mass, ${ }^{P} \mathbf{I}_{G}$ is the inertia matrix of the platform about $G$ in the $P x^{\prime} y^{\prime} z^{\prime}$ coordinate system, and $\mathbf{I}_{G}=\mathbf{R}^{P} \mathbf{I}_{G} \mathbf{R}^{T}$ is the platform inertia matrix in the fixed system $O x y z$. Vectors $\mathbf{f}_{C}$ and $\mathbf{m}_{C}$ are the resultant forces and moments of all forces transmitted by the cables to the mobile platform. If $T_{i}$ is the tension force in the $i$-th cable and $\tau_{i}=T_{i} /\left\|\boldsymbol{\rho}_{i}\right\|$, then:

$$
\left[\begin{array}{c}
\mathbf{f}_{C} \\
\mathbf{m}_{C}
\end{array}\right]=-\mathbf{J}_{v}^{T} \boldsymbol{\tau}
$$

where $\boldsymbol{\tau}=\left[\tau_{1}, \ldots, \tau_{n}\right]^{T}$.
Equations 32 and 33 can be expressed in compact form as (see Appendix A):

$$
\mathbf{M} \ddot{\mathbf{q}}-\mathbf{s}-\mathbf{B} \boldsymbol{\tau}=\mathbf{0}
$$

where:

$$
\begin{gathered}
\mathbf{M}=\left[\begin{array}{cc}
m \mathbf{I}_{3} & -m \tilde{\mathbf{r}}^{\prime} \mathbf{H} \\
-m \mathbf{H}^{T} \tilde{\mathbf{r}}^{\prime T} & \mathbf{H}^{T} \mathbf{I}_{P} \mathbf{H}
\end{array}\right] \\
\mathbf{I}_{P}=\mathbf{I}_{G}-m \tilde{\mathbf{r}}^{\prime} \tilde{\mathbf{r}}^{\prime} \\
\mathbf{B}=-\mathbf{J}_{q}^{T} \\
\mathbf{s}=\left[\begin{array}{c}
m\left(\tilde{\mathbf{r}}^{\prime} \dot{\mathbf{H}}+\tilde{\boldsymbol{\omega}} \tilde{\mathbf{r}}^{\prime} \mathbf{H}\right) \dot{\boldsymbol{\epsilon}}+\mathbf{f}_{G} \\
\mathbf{H}^{T}\left[-\left(\mathbf{I}_{P} \dot{\mathbf{H}}+\tilde{\boldsymbol{\omega}} \mathbf{I}_{P} \mathbf{H}\right) \dot{\boldsymbol{\epsilon}}+\mathbf{m}_{G}+\tilde{\mathbf{r}}^{\prime} \mathbf{f}_{G}\right]
\end{array}\right]
\end{gathered}
$$

and $\mathbf{I}_{3}$ is the $3 \times 3$ identity matrix.

In the case of an underactuated system, the generalized coordinates can be partitioned in $n$ actuated coordinates $\mathbf{q}_{a}$ and $\lambda=h-n$ unactuated coordinates $\mathbf{q}_{u}$, depending on the task that the platform is required to perform. The actuated coordinates $\mathbf{q}_{a}$ can be fully assigned, whereas the unactuated ones $\mathbf{q}_{u}$ will be determined by the dynamics of the system. According to the aforementioned partition, Eq. 35) can be rewritten as:

$$
\left[\begin{array}{ll}
\mathbf{M}_{a a} & \mathbf{M}_{a u} \\
\mathbf{M}_{u a} & \mathbf{M}_{u u}
\end{array}\right]\left[\begin{array}{c}
\ddot{\mathbf{q}}_{a} \\
\ddot{\mathbf{q}}_{u}
\end{array}\right]-\left[\begin{array}{l}
\mathbf{s}_{a} \\
\mathbf{s}_{u}
\end{array}\right]-\left[\begin{array}{l}
\mathbf{B}_{a} \\
\mathbf{B}_{u}
\end{array}\right] \boldsymbol{\tau}=\mathbf{0}
$$

We can then isolate $\ddot{\mathbf{q}}_{u}$ as:

$$
\ddot{\mathbf{q}}_{u}=\mathbf{M}_{u u}^{-1}\left(\mathbf{s}_{u}+\mathbf{B}_{u} \boldsymbol{\tau}-\mathbf{M}_{u a} \ddot{\mathbf{q}}_{a}\right)
$$

Substituting $\ddot{\mathbf{q}}_{u}$ in the remaining relations of Eq. 40 yields the normalized cable tensions as functions of the imposed motion profile $\mathbf{q}_{a}(t)$, thus allowing slackness avoidance to be verified as:

$$
\boldsymbol{\tau}=\left(\mathbf{B}_{a}-\mathbf{M}_{a u} \mathbf{M}_{u u}^{-1} \mathbf{B}_{u}\right)^{-1} \boldsymbol{\mu}
$$

where:

$$
\boldsymbol{\mu}=\left(\mathbf{M}_{a a}-\mathbf{M}_{a u} \mathbf{M}_{u u}^{-1} \mathbf{M}_{u a}\right) \ddot{\mathbf{q}}_{a}+\mathbf{M}_{a u} \mathbf{M}_{u u}^{-1} \mathbf{s}_{u}-\mathbf{s}_{a}
$$

From an analytical standpoint, we can substitute Eq. (42) in (41) and obtain a completely differential system in the actuated and unactuated coordinates. This system represents the second-order non-holonomic constraint, also known as the internal dynamics, arising from the underactuated nature of the system [33].

\section{Rest-to-Rest Trajectory PlanNing}

System theory defines the problem of rest-to-rest trajectory planning for an underactuated mechanical system as a transition problem between stationary setpoints [34]. Such a transition has been proven to be possible [35], in most cases, if the system undergoes an additional pre-actuation or post-actuation phase, that is, if the system is actuated when $t<0, t>T$ (where $T$ is the transition time) or both. However, this leads to a theoretical impossibility of bringing the system at rest in a predefined time. In addition, a precise path tracking can not be ensured, as the uncontrolled coordinates behavior cannot be predicted, possibly leading the system to instability (i.e. to an oscillatory behavior), thus strongly limiting practical applications. 
In [36], a new method was proposed for the trajectory design of Single-Input Single-Output (SISO) systems. The same approach was extended to Multi-Input Multi-Output (MIMO) systems in [37, and later to systems performing not only single transitions, but also cyclic tasks [38. These methods ensure that the system is brought to a stationary position in a prescribed time $T$, but the nominal trajectory of the actuated coordinates undergoes a substantial modification. This is often not desirable (when not dangerous) in industrial applications involving robots, because of possible interference with obstacles.

In this section, the novel trajectory planning method introduced in [1] is explained in detail. This method avoids a geometric modification of the path that the actuated coordinates are supposed to track, focusing instead on a specific design of the motion law that describes how these coordinate evolve along the path itself. The design of such a motion law relies on the solution of a $B V P$, formulated as the problem of finding a solution to the differential equation (41), with constraints on position and velocity at start and end times. A heuristic solution strategy for the specific $B V P$ at hand is proposed in Section IV-C.

\section{A. Formulation of the Problem}

When planning the trajectory of a completely-actuated manipulator, we may define both the geometric path of a reference point on the end-effector and the orientation of the latter. If the end-effector is underactuated, however, only a subset $\mathbf{q}_{a}$ of its generalized coordinates may be assigned. It is convenient to consider a parametric representation of the path to track, such as $\mathbf{q}_{a}=\mathbf{q}_{a}(u(t))$. We refer to the parameter $u(t)$ as the motion law, which is a function of time, with initial and final conditions $u(0)=0$ and $u(T)=1$. The composition $\left(\mathbf{q}_{a} \circ u\right)=\mathbf{q}_{a}(u(t))$ is what is usually referred to as the trajectory.

A variety of methods may be employed to design the motion law. In the case of a completely or redundantly actuated manipulator the problem of a stationary set point change only requires the solution of a system of linear equations emerging from the fulfilment of some boundary conditions and the necessity of a continuous and differentiable function. Polynomial motion laws are often sufficient to satisfy start- and end-point conditions. An easy way to devise such polynomials is to use so-called transition polynomials [39] of degree $2 r+1$ :

$$
u(t)=\sum_{i=r+1}^{2 r+1} a_{i}\left(\frac{t}{T}\right)^{i}, \quad t \in[0, T]
$$

where coefficients $a_{i}$ 's do not depend on the task at hand and are given by:

$$
a_{i}=\frac{(-1)^{i-r-1}(2 r+1) !}{i \cdot r !(i-r-1) !(2 r+1-i) !}
$$

with (if $r \geq 2$ ):

$$
\sum_{i=r+1}^{2 r+1} a_{i}=1, \quad \sum_{i=r+1}^{2 r+1} i a_{i}=0, \quad \sum_{i=r+1}^{2 r+1} i(i-1) a_{i}=0
$$

The index $r$ stands for the maximum order of derivation up to which the continuity of the polynomial is required.

This approach is rarely sufficient for rest-to-rest motions if the system is underactuated, because it does not take into account the internal dynamics (cf. Eq. (41)). In fact, depending on the inertial effect caused by the geometric path and the chosen motion law, the uncontrolled coordinates $\mathbf{q}_{u}$ may not reach a stationary condition when the end point is attained.

In order to achieve the desired result, the nonholonomic constraint in Eq. 41) must be considered in the planning phase. The stationary conditions on $\mathbf{q}_{u}$ in the start- and end-points can be regarded as boundary conditions (BCs) for the differential equation [4], thus leading to a $B V P$. This problem has generally no solution when both the path $\mathbf{q}_{a}(u)$ and the motion law $u(t)$ are assigned. On the other hand, if modifications of $u(t)$ are allowed, the problem may admit a solution.

In order to numerically solve the differential equation 41), we first express it in state form:

$$
\begin{gathered}
\mathbf{x}=\left[\begin{array}{c}
\mathbf{q}_{u} \\
\dot{\mathbf{q}}_{u}
\end{array}\right] \\
\dot{\mathbf{x}}=\left[\begin{array}{c}
\dot{\mathbf{q}}_{u} \\
\mathbf{M}_{u u}^{-1}\left(\mathbf{s}_{u}+\mathbf{B}_{u} \boldsymbol{\tau}-\mathbf{M}_{u a} \ddot{\mathbf{q}}_{a}\right)
\end{array}\right]=\mathbf{f}\left(\mathbf{x}, \mathbf{q}_{a}, \dot{\mathbf{q}}_{a}, \ddot{\mathbf{q}}_{a}\right)
\end{gathered}
$$

where $\boldsymbol{\tau}$ is given by Eq. 42.

For a rest-to-rest trajectory planning, $B C s$ are:

$$
\mathbf{x}(0)=\left[\begin{array}{c}
\mathbf{q}_{u}\left(\mathbf{q}_{a}(0)\right) \\
\mathbf{0}
\end{array}\right]:=\mathbf{x}_{0}, \quad \mathbf{x}(T)=\left[\begin{array}{c}
\mathbf{q}_{u}\left(\mathbf{q}_{a}(T)\right) \\
\mathbf{0}
\end{array}\right]:=\mathbf{x}_{T}
$$

where $\left[\mathbf{q}_{a}(0)^{T} \mathbf{q}_{u}\left(\mathbf{q}_{a}(0)\right)^{T}\right]^{T}$ and $\left[\mathbf{q}_{a}(T)^{T} \mathbf{q}_{u}\left(\mathbf{q}_{a}(T)\right)^{T}\right]^{T}$ are stable equilibrium configurations of the system, that can be obtained as in [3], 24, 25.

Equation (48) has dimension $2 \lambda$ and can only match $2 \lambda$ out of the $4 \lambda B C s$ established in (49). One way to provide a solution to the problem is to consider $2 \lambda$ additional scalar parameters $\kappa_{1}, \ldots, \kappa_{2 \lambda}$ (called free parameters), so that $\mathbf{q}_{a}=$ $\mathbf{q}_{a}(\boldsymbol{\kappa}, t)$. The BVP with free parameters becomes then:

$$
\left\{\begin{array}{l}
\dot{\mathbf{x}}=\mathbf{f}\left(\mathbf{x}(\boldsymbol{\kappa}, t), \mathbf{q}_{a}(\boldsymbol{\kappa}, t), \dot{\mathbf{q}}_{a}(\boldsymbol{\kappa}, t), \ddot{\mathbf{q}}_{a}(\boldsymbol{\kappa}, t)\right) \\
\mathbf{x}(0)=\mathbf{x}_{0}, \quad \mathbf{x}(T)=\mathbf{x}_{T}
\end{array}\right.
$$

The solution of 50 is a set $\{\boldsymbol{\kappa}, \mathbf{x}(\boldsymbol{\kappa}, t)\}$, where the vector of free parameters $\boldsymbol{\kappa} \in \mathbb{R}^{2 \lambda}$ is calculated so as the $B C$ in Eq. [50) are satisfied.

\section{B. Modification of the Motion Law}

In the case of a constrained trajectory geometric path, the motion law is the only element that can undergo a modification, that is, $\mathbf{q}_{a}=\mathbf{q}_{a}(u(\boldsymbol{\kappa}, t))[40$. One way to design such a modified motion law $u$, so that the actuated coordinates can meet the start and end conditions prescribed by the task, is to consider the composition $(u \circ \gamma)(\boldsymbol{\kappa}, t)=u(\gamma(\boldsymbol{\kappa}, t))$, now expressed as:

$$
\begin{aligned}
& u(\gamma(\boldsymbol{\kappa}, t))=\sum_{i=r+1}^{2 r+1} a_{i} \gamma^{i}(\boldsymbol{\kappa}, t) \\
& \gamma(\boldsymbol{\kappa}, 0)=0, \quad \gamma(\boldsymbol{\kappa}, T)=1, \quad \forall \boldsymbol{\kappa} \in \mathbb{R}
\end{aligned}
$$


where $a_{i}$ is still expressed as in 45 and the function $\gamma(\boldsymbol{\kappa}, t)$ is continuous and differentiable up to the second order.

For the purpose of this article, $r=3$, so that continuity of jerk can be imposed in the start and end positions, and any discontinuity in the cable tensions $\boldsymbol{\tau}$ can be (at least theoretically) avoided. In this way, we try to eliminate a different potential source of residual oscillations.

As an example, $\gamma(\boldsymbol{\kappa}, t)^{3}$ may be intuitively designed as a polynomial of order $2 \lambda+1$ :

$$
\gamma(\boldsymbol{\kappa}, t)=\alpha t+\sum_{i=2}^{2 \lambda+1} \kappa_{i-1} t^{i}, \quad \alpha=\frac{1-\sum_{i=2}^{2 \lambda+1} \kappa_{i-1} T^{i}}{T}
$$

Accordingly, the time derivative of the actuated coordinates can be expressed as:

$$
\dot{\mathbf{q}}_{a}=\frac{\partial \mathbf{q}_{a}}{\partial u} \frac{\partial u}{\partial \gamma} \frac{\partial \gamma}{\partial t}=\mathbf{q}_{a}^{\prime} u^{*} \dot{\gamma}
$$

where $(\cdot)^{\prime}$ denotes the partial derivative with respect to $u$ and $(\cdot)^{*}$ the partial derivative with respect to $\gamma$. The secondorder time derivative is hence:

$$
\ddot{\mathbf{q}}_{a}=\mathbf{q}_{a}^{\prime \prime}\left(u^{*} \dot{\gamma}\right)^{2}+\mathbf{q}_{a}^{\prime}\left(u^{*} \ddot{\gamma}+u^{* *} \dot{\gamma}^{2}\right)
$$

It should be noted that, as long as conditions [52 are satisfied and $r \geq 2$, no other conditions have to be imposed on $\gamma(\boldsymbol{\kappa}, t)$. In fact, $\dot{\mathbf{q}}_{a}(\boldsymbol{\kappa}, T)=0$ and $\ddot{\mathbf{q}}_{a}(\boldsymbol{\kappa}, T)=0$ is ensured for any $\boldsymbol{\kappa}$ by $u^{*}(\gamma(\boldsymbol{\kappa}, T))=0$ and $u^{* *}(\gamma(\boldsymbol{\kappa}, T))=0$. Other formulations for either $\gamma(\boldsymbol{\kappa}, t)$ or the whole $u(\boldsymbol{\kappa}, t)$ may be employed in order to achieve similar results. From a practical point of view, though, the choice of $\gamma(\boldsymbol{\kappa}, t)$ can affect the convergence rate and speed of the solution algorithm described in Section IV-C In addition, depending on the specific formulation of $\gamma(\boldsymbol{\kappa}, t)$, it may not be easy to determine an initial guess for $\boldsymbol{\kappa}$, which is needed in the numerical solution of Eq. [50).

\section{Solution of the BVP with Free Parameters}

Free parameters $\boldsymbol{\kappa}$ can be found as a consequence of the numerical solution of the BVP expressed by Eq. [50). A number of algorithms are proposed in the literature and even implemented in commercial softwares, such as the bup $4 c$ and bup5c routines available in any MATLAB distribution 41. These algorithms are finite-difference codes that implement a collocation formula [42] and, thus, require a suitable set-up in order to work efficiently and find a solution within a reasonable tolerance. However, even in this case, there is still no guarantee of success.

During our simulation campaign, we were not able to solve problem [50) by employing these standard methods, thus we heuristically formulate the problem as a combination of an Initial Value Problem (IVP) followed by the solution of a system of nonlinear equations. This approach is similar to a classic iterative shooting method [42, which is

${ }^{3} \mathrm{~A}$ general formulation for $\gamma(\mathbf{k}, t)$ is given by:

$$
\gamma(\boldsymbol{\kappa}, t)=\frac{t}{T}\left[\left(1-\frac{t}{T}\right) h(\boldsymbol{\kappa}, t)+1\right]
$$

where $h(\boldsymbol{\kappa}, t)$ is any continuous and differentiable function up to the second order. a state-of-the-art method for the solution of standard BVPs without free parameters: at each iteration of the algorithm, the value of the free parameters is modified instead of the problem initial conditions. For any assigned $\boldsymbol{\kappa}$, let $\mathbf{x}(\boldsymbol{\kappa}, t)$ be the solution of the IVP defined by:

$$
\left\{\begin{array}{l}
\dot{\mathbf{x}}=\mathbf{f}\left(\mathbf{x}(\boldsymbol{\kappa}, t), \mathbf{q}_{a}(\boldsymbol{\kappa}, t), \dot{\mathbf{q}}_{a}(\boldsymbol{\kappa}, t), \ddot{\mathbf{q}}_{a}(\boldsymbol{\kappa}, t)\right) \\
\mathbf{x}(0)=\mathbf{x}_{0}
\end{array}\right.
$$

In general, for an arbitrary $\boldsymbol{\kappa}, \mathbf{x}(\boldsymbol{\kappa}, t)$ does not meet the end-point condition in Eq. [50, namely $\mathbf{x}(T) \neq \mathbf{x}_{T}$. Consider then the nonlinear equation in the unknown $\boldsymbol{\kappa}$ defined by:

$$
\mathbf{F}(\boldsymbol{\kappa})=\mathbf{x}(\boldsymbol{\kappa}, T)-\mathbf{x}_{T}=\mathbf{0}
$$

A solution for equation (57) may be found by the following iterative procedure:

0 ) assign $\boldsymbol{\kappa}_{i}$ for $i=0$, e.g. $\boldsymbol{\kappa}_{i}=\mathbf{0}$, and establish an adequately small tolerance $\zeta \in \mathbb{R}$;

1) evaluate $\mathbf{x}\left(\boldsymbol{\kappa}_{i}, T\right)$ as the end-point of the solution $\mathbf{x}\left(\boldsymbol{\kappa}_{i}, t\right)$ of the IVP [56).

2) If $\left\|\mathbf{F}\left(\boldsymbol{\kappa}_{i}\right)\right\| \leq \zeta,\left\{\boldsymbol{\kappa}_{i}, \mathbf{x}\left(\boldsymbol{\kappa}_{i}, t\right)\right\}$ is a solution of the $B V P$ [50], otherwise set $\boldsymbol{\kappa}_{i+1}=\boldsymbol{\kappa}_{i}+\mathbf{J}_{F}^{-1}\left(\boldsymbol{\kappa}_{i}\right) \mathbf{F}\left(\boldsymbol{\kappa}_{i}\right)$ and repeat the iteration.

$\mathbf{J}_{F}(\boldsymbol{\kappa})=\partial \mathbf{F} / \partial \boldsymbol{\kappa}$ is the Jacobian of Eq. 57) with respect to $\boldsymbol{\kappa}$ and it can be approximated by finite differences at every iteration. Finite-difference Jacobian can be efficiently computed by using several parallel threads. However, due to the iterative and approximated nature of the algorithm, the maximum computational time cannot be predicted in advance. Accordingly, this algorithm is not suitable for realtime computation, and has to be employed offline. In our simulations, the algorithm has proven to be sub-linearly convergent in the case it is started from a generic initial guess for $\boldsymbol{\kappa}$.

Once a solution $\{\boldsymbol{\kappa}, \mathbf{x}(\boldsymbol{\kappa}, t)\}$ is found, the trajectory $\mathbf{q}_{a}(\boldsymbol{\kappa}, t)$ may be computed, and the cable total length is found according to Eq. (16) as:

$$
l_{i}(t)=\sqrt{\boldsymbol{\rho}_{i}^{T} \boldsymbol{\rho}_{i}}+r_{i}\left(\pi-\psi_{i}\right), \quad i=1, \ldots, n
$$

No explicit constraints on cable tensions or motor torques are considered in this work other than positive cable tensions, which are verified during the integration of eq. 56) via Eq. $42^{4}$. In addition, it should be noted that the assigned transition time $T$ cannot be arbitrarily low. It is outside the scope of this paper to determine an optimal transition time for assigned set-points. However, in all simulations and experiments conducted with the prototype described in Section V] we heuristically determined that a transition time resulting from an average speed of the platform reference point of approximatly $1 \mathrm{~m} / \mathrm{s}$ between assigned set-points always results in a solution of 57) with positive tensions in all cables.

\footnotetext{
${ }^{4}$ While iteratively solving Eq. [57, the IVP [56] is integrated at each step, and the values of $\boldsymbol{\tau}(t)$ are calculated by Eq. (42) for an assigned $\boldsymbol{\kappa}_{i}$. In the case a negative value of one tension is determined, the IVP integration is re-initialized with a different value of $\boldsymbol{\kappa}_{i}$
} 


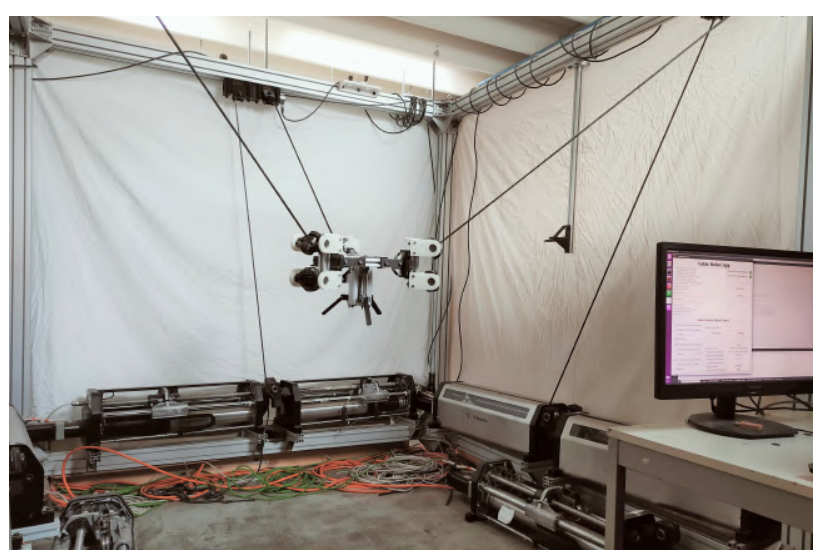

Figure 4. Prototype overview.

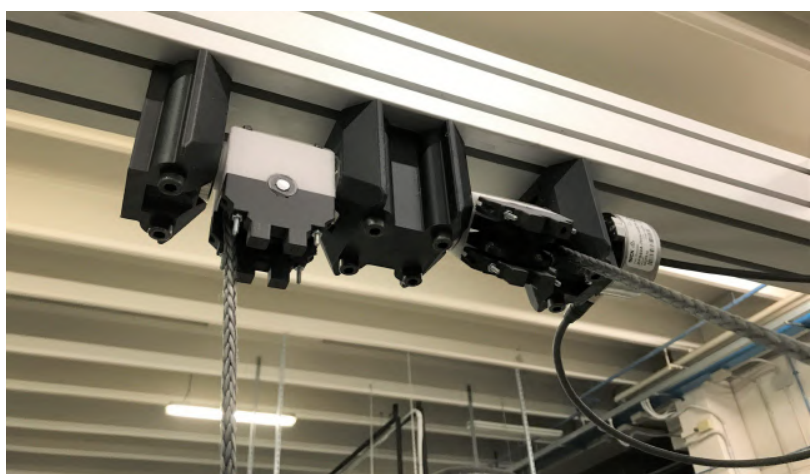

Figure 5. Prototype swivel pulleys.

\section{EXPERIMENTAL VALIDATION}

The trajectory-planning methodology considered in this paper has been implemented in a MATLAB code. For every desired $s$-th transition between stationary set points, the inputs needed by the trajectory-planner are:

- the transition time ${ }^{s} T$;

- the transition set-points (namely ${ }^{s-1} \mathbf{q}_{a}$ and ${ }^{s} \mathbf{q}_{a}$ );

- the transition geometric path parametrization ${ }^{s} \mathbf{q}_{a}(u)$;

After the solution of the problem defined by Eq. [50], the outputs of the planning routine are:

- the array of free parameters ${ }^{s} \boldsymbol{\kappa}$;

- the actuated and unactuated coordinates, that is, ${ }^{s} \mathbf{q}_{a}(t)$ and ${ }^{s} \mathbf{q}_{u}(t)$ for $t \in\left[0,{ }^{s} T\right]$;

- the subsequent cable lengths ${ }^{s} l_{i}(t)(i=1, \cdots, n)$ for $t \in\left[0,{ }^{s} T\right]$, determined by [58.

In the following, two scenarios will be addressed: $i$ ) a 6-DoF spatial CDPR actuated by 3 cables whose reference point must track consecutive line segments; $i$ ) the same spatial robot as in case $(i)$ whose reference point must track consecutive circular arcs.

In the preliminary conference version of this paper 11 our trajectory-planning method was tested on a 3-DoF planar CDPR actuated by 2 cables. The kinematics and dynamics of such a robot are greatly simplified by the planar motion (and thus by the limited available freedoms) and by fixed cable exit points from the frame. This leads to a very fast and easier solution of the $B V P$ defined by
Table I

ACTUATION UNIT PROPERTIES

\begin{tabular}{|c|c|c|c|}
\hline$i$ & 1 & 2 & 3 \\
\hline $\mathbf{d}_{i}[\mathrm{~m}]$ & {$\left[\begin{array}{c}0.160 \\
-0.835 \\
-0.025\end{array}\right]$} & {$\left[\begin{array}{c}2.175 \\
0.180 \\
-0.035\end{array}\right]$} & {$\left[\begin{array}{c}0.260 \\
1.290 \\
-0.043\end{array}\right]$} \\
\hline$r_{i}[\mathrm{~m}]$ & 0.025 & 0.025 & 0.025 \\
\hline$P_{\mathbf{a}_{i}^{\prime}[\mathrm{m}]}$ & {$\left[\begin{array}{c}0 \\
-0.267 \\
0.270\end{array}\right]$} & {$\left[\begin{array}{c}0.231 \\
0.133 \\
0.270]\end{array}\right]$} & {$\left[\begin{array}{c}-0.231 \\
0.133 \\
0.270\end{array}\right]$} \\
\hline $\mathbf{x}_{i}$ & $\mathbf{j}$ & $-\mathbf{i}$ & $-\mathbf{j}$ \\
\hline $\mathbf{y}_{i}$ & $-\mathbf{i}$ & $-\mathbf{j}$ & $\mathbf{i}$ \\
\hline $\mathbf{z}_{i}$ & $\mathbf{k}$ & $\mathbf{k}$ & $\mathbf{k}$ \\
\hline
\end{tabular}

Table II

PlATFORM PROPERTIES

\begin{tabular}{|c|ccc|}
\hline$m[\mathrm{Kg}]$ & $\mathbf{I}_{G}\left[\mathrm{Kg} \cdot \mathrm{m}^{2}\right]$ & ${ }^{P} \mathbf{r}^{\prime}[\mathrm{m}]$ \\
\hline \multirow{3}{*}{8} & {$\left[\begin{array}{ccc}0.14 & 0 & 0 \\
0 & 0.14 & 0 \\
0 & 0 & 0.216\end{array}\right]$} & {$\left[\begin{array}{c}0 \\
0 \\
0.182\end{array}\right]$} \\
\hline
\end{tabular}

Eq. 50. The details of the model and the experimental results are available in [1]. They are not reported here due to space limitations, since they do not add anything particularly relevant to what will be presented for the spatial robot. The geometrical and inertial properties of the 6DoF spatial prototype are summarized in Tables II and II where $\mathbf{i}=[1 ; 0 ; 0]]^{T}, \mathbf{j}=[0 ; 1 ; 0]^{T}$ and $\mathbf{k}=[0 ; 0 ; 1]^{T}$. The robot platform and swivel pulleys are portrayed in Figs. 4 and 5.

The platform reference point $P$ will transit and rest in 4 set-points. The stationary (stable) poses of the platform, evaluated as in [3, 24], 25], are:

$$
{ }^{0} \mathbf{q}=\left[\begin{array}{c}
1.596 \\
0.183 \\
-1.300 \\
-0.050 \\
-0.603 \\
-0.575
\end{array}\right] \quad{ }^{1} \mathbf{q}=\left[\begin{array}{c}
1.165 \\
0.211 \\
-0.900 \\
-0.005 \\
-0.210 \\
-0.556
\end{array}\right] \quad{ }^{2} \mathbf{q}=\left[\begin{array}{c}
0.587 \\
0.222 \\
-1.300 \\
0.009 \\
0.255 \\
-0.562
\end{array}\right] \quad{ }^{3} \mathbf{q}={ }^{0} \mathbf{q}
$$

and the transition times are:

$$
{ }^{1} T=1.5 \mathrm{~s} \quad{ }^{2} T=1.5 \mathrm{~s} \quad{ }^{3} T=2 \mathrm{~s}
$$

Each transition is separated from the next one by a pause of $5 \mathrm{~s}$, so that potential residual oscillations of the platform can be highlighted. "Rest-to-Rest" (RTR) trajectories, designed according to our approach, are compared with "Standard" $(S T D)$ trajectories, which are defined such as:

$$
\boldsymbol{\kappa}=\mathbf{0}, \quad \gamma(\boldsymbol{\kappa}, t)=\frac{t}{T}
$$

In this case, the end-effector orientation is estimated by forward integration of Eq. (56) and the cable lengths by Eq. (58). It should be noted that the uncontrolled coordinates $\mathbf{q}_{u}(t)$ of the $e-e$, and thus the lengths of the cables, are generally different for distinct values of $\boldsymbol{\kappa}$. Because of this, the equilibrium poses of the $e-e$ in the STD and RTR cases 


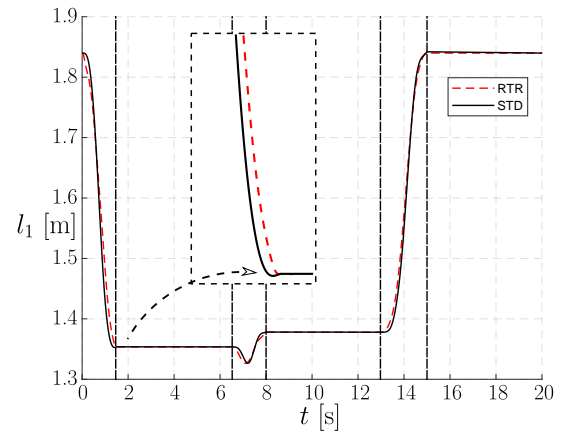

(a) First cable

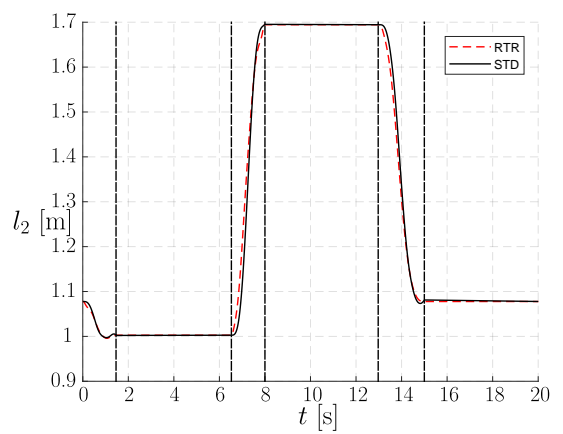

(b) Second cable

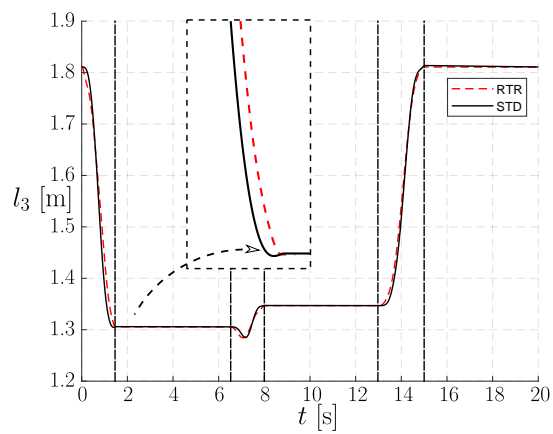

(c) Third cable

Figure 6. Actuator position set-points for RTR and STD trajectories, in the case of linear paths. Transitions between set-points are delimited by vertical dashed lines

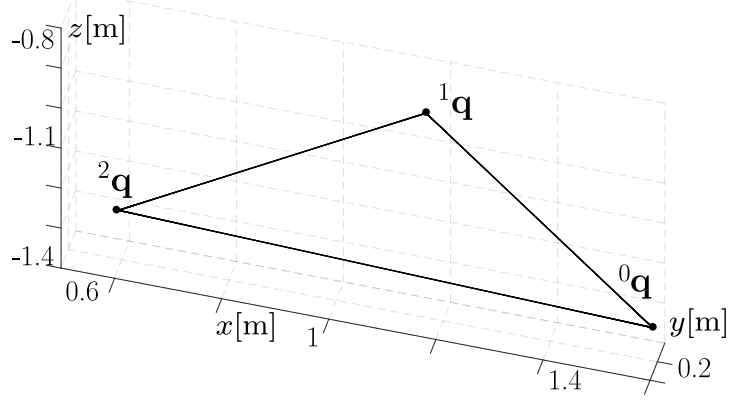

Figure 7. Straight line paths.

will also be different. In order to be able to compare different subsequent trajectories, STD cable lengths were quasistatically varied during the pause time in order to match the RTR ones, and thus have the same start configuration. For the transitions considered in this paper, the difference between them is in the order of some millimiters, so that this procedure does not amplify residual oscillations (as it can be see in Fig. 8 and 13 where the procedure was simulated).

1) Straight Line Trajectories: In the first example, the set-points are connected by linear paths (Fig. 7). The $s$-th transition is parametrized as:

$$
{ }^{s} \mathbf{p}(u)={ }^{s-1} \mathbf{p}+\left({ }^{s} \mathbf{p}-{ }^{s-1} \mathbf{p}\right) u
$$

By employing the method outlined in Section IV-C the solution of the $B V P$ defined by Eq. (50) for $s=1,2,3$ is found starting from an initial guess $\boldsymbol{\kappa}=\mathbf{0}_{6 \times 1}$ in averagely $1.5 \mathrm{~min}$ by using a MATLAB implementation installed on a Windows $10 \mathrm{PC}$, with a 7th generation Intel I7 CPU and $16 \mathrm{~Gb}$ of RAM. The results are summarized in Eq. 61] and Figs. 6 and 8 .

$$
{ }^{1} \boldsymbol{\kappa}=\left[\begin{array}{c}
-14.006 \\
41.906 \\
-67.565 \\
60.146 \\
-27.779 \\
5.195
\end{array}\right] \quad{ }^{2} \boldsymbol{\kappa}=\left[\begin{array}{c}
-12.278 \\
38.731 \\
-66.101 \\
61.907 \\
-29.927 \\
5.827
\end{array}\right] \quad{ }^{3} \boldsymbol{\kappa}=\left[\begin{array}{c}
-4.204 \\
8.826 \\
-10.157 \\
6.559 \\
-2.234 \\
0.312
\end{array}\right]
$$

In order to verify the effectiveness of the proposed trajectory planning, no feedback on the platform actual pose was used to stabilize or correct the end-effector position and orientation during experimental testing. A visual result of the experimentation can be found in the video file attached to this paper. Quantitative results regarding the end-effector pose can be found in Fig. 9. Since no external measurement system, such as a laser tracker, was available in our laboratory during the experimental campaign, an indirect approach was used. Angles $\sigma_{1}, \sigma_{2}$ and $\sigma_{3}$ were measured by incremental encoders attached to the swivel pulleys' axes. By employing such measurements and the commanded cable lengths, the pose of the platform was estimated making use of a direct kinematic algorithm that employs additional cable orientation measurements 43.

This approach does not allow the platform pose to be inferred with a high precision during a dynamic motion (on the one hand, cables can slightly oscillate in the grooves of their pulleys due to clearance; on the other, pulleys swivel with some lag compared to the theoretical kinematic model, mainly due to friction in the mechanical transmission chains). However, it provides a simple means to effectively compare $R T R$ and $S T D$ trajectories. It is apparent that, though the actuator set-points are very similar for both trajectories, the results in terms of end-effector pose are significantly different. The difference in the global motion of the platform can be explained, in general, by considering that limited difference in the position set-points may be associated with large difference in their higher order derivatives, which play a key role in the dynamics of the system. STD trajectories display residual oscillations which are damped over time by dissipative effects and a slight drift in the $e-e$ coordinates, which may be attributed to cables oscillating into the pulley grooves. On the other hand, RTR trajectories results do not show any significant oscillation in the measurements provided by our feedback system (Figs. 10a and 10b show an enhanced view of the most critical oscillations observed during experiments, for $8 \mathrm{~s}<t<13 \mathrm{~s}$ ). We can clearly see that both position and angular $e-e$ coordinates remain constant after the end of the second transition when RTR trajectories are used. The video of the experimentation also displays little to no oscillation left after $R T R$ transitions. The small residual swinging of the platform at the end of transitions is due to 


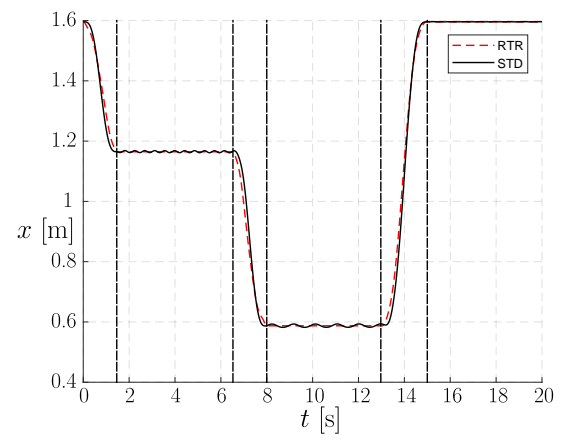

(a) $x$ coordinate

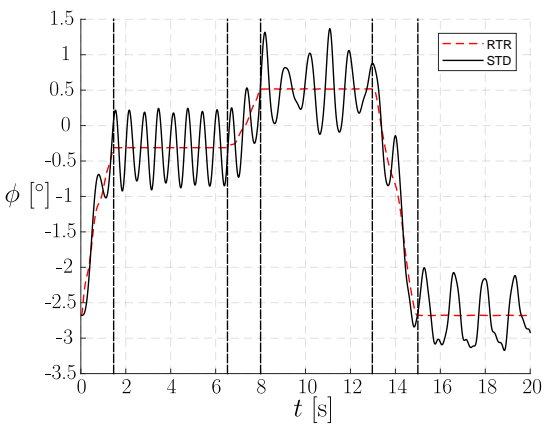

(d) $\phi$ Euler angle

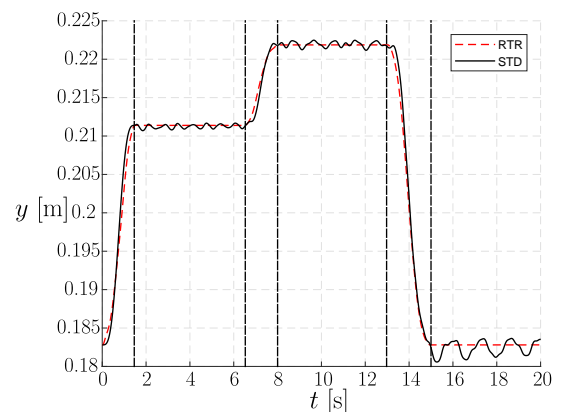

(b) $y$ coordinate

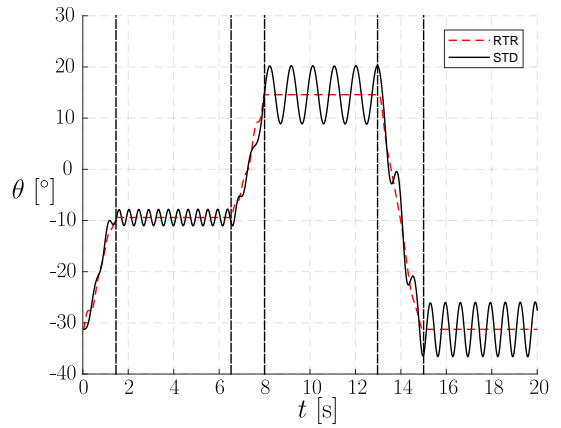

(e) $\theta$ Euler angle

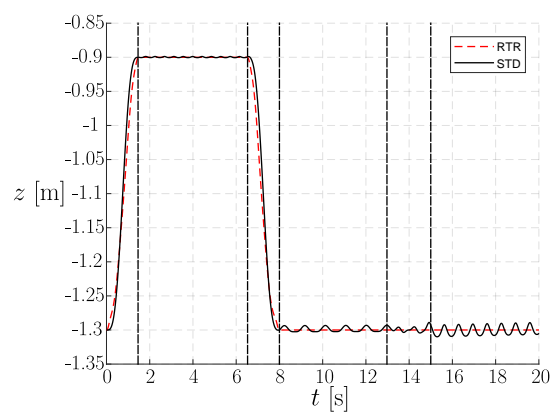

(c) $z$ coordinate

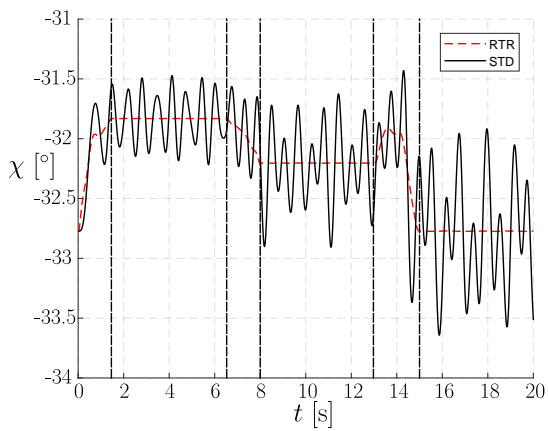

(f) $\chi$ Euler angle

Figure 8. Computed end-effector pose for RTR and STD trajectories, in the case of linear paths. Transitions between set-points are delimited by vertical dashed lines

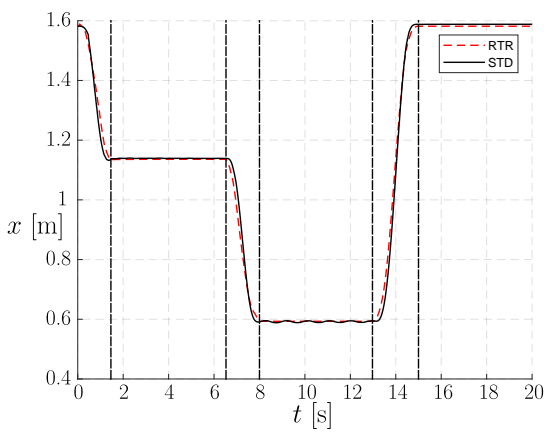

(a) $x$ coordinate

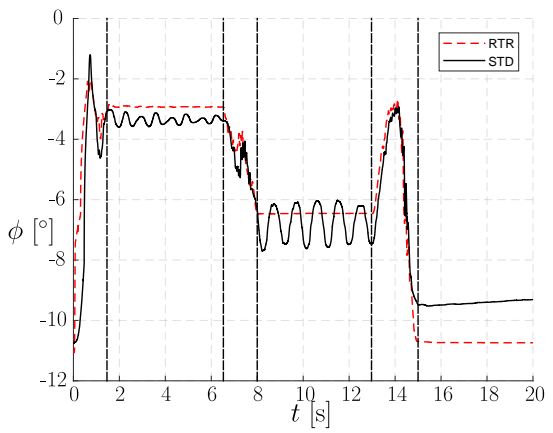

(d) $\phi$ Euler angle

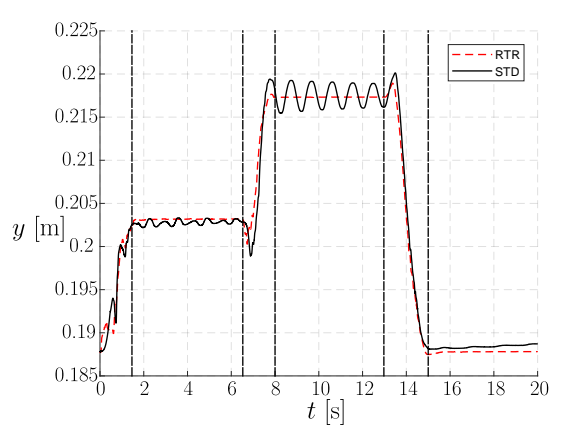

(b) $y$ coordinate

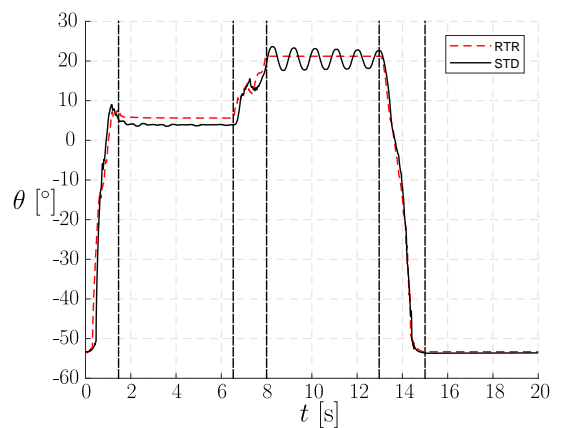

(e) $\theta$ Euler angle

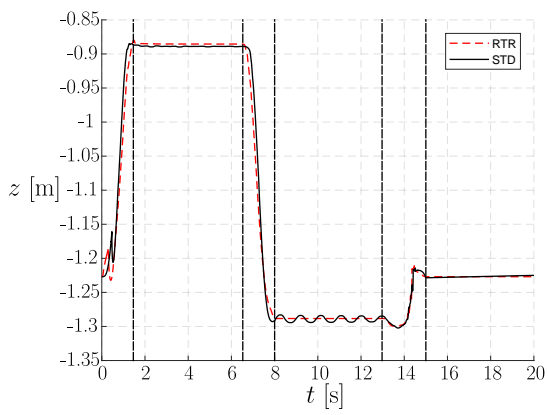

(c) $z$ coordinate

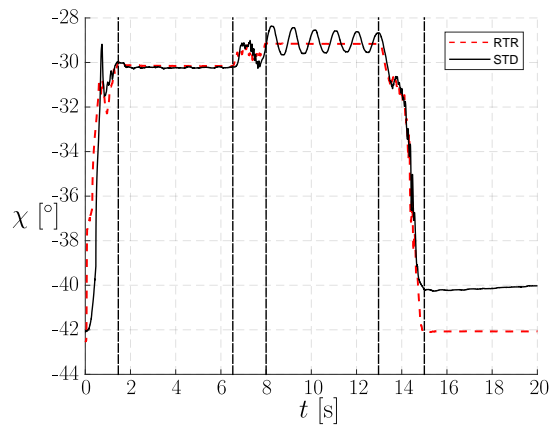

(f) $\chi$ Euler angle

Figure 9. Measured end-effector pose for RTR and STD trajectories, in the case of linear paths. Transitions between set-points are delimited by vertical dashed lines

minor-importance phenomena that are not included in the dynamic model, such as clearance, elasticity, friction, etc.

Also, it can be noted from the planning results (Figs. 6a and $6 \mathrm{c}$ that, in the first transition, the slope of the cable commanded length in the RTR case is steeper compared to the one of the $S T D$ case. Even though the encoder measurement of our prototype motors show no tracking error, frictional effects in the cable transmission could have 


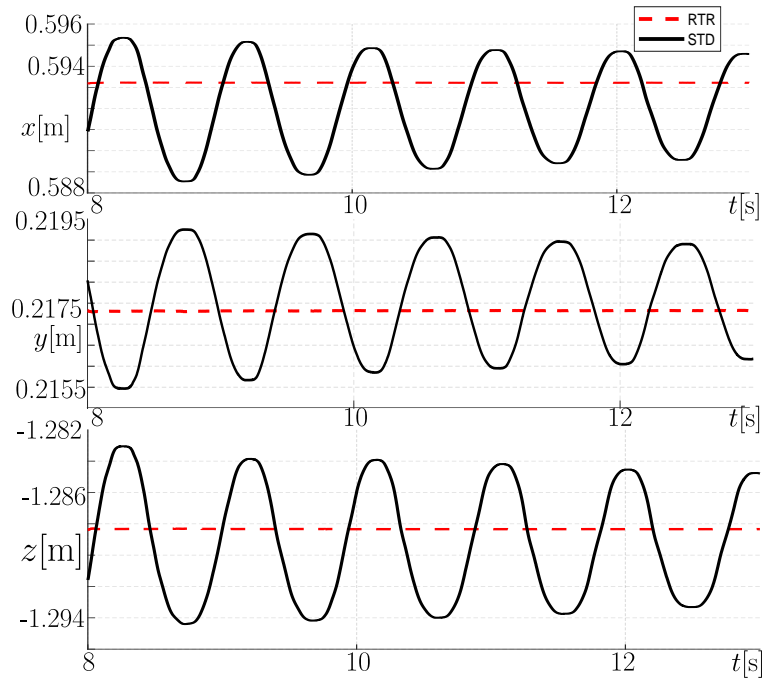

(a) End-effector reference position

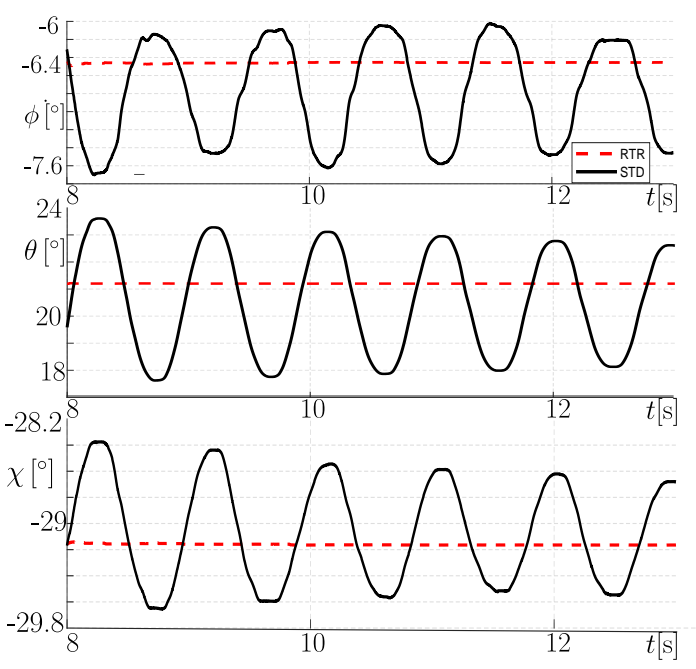

(b) End-effector Euler angles

Figure 10. Enhanced view of most critical measured oscillations during linear paths.

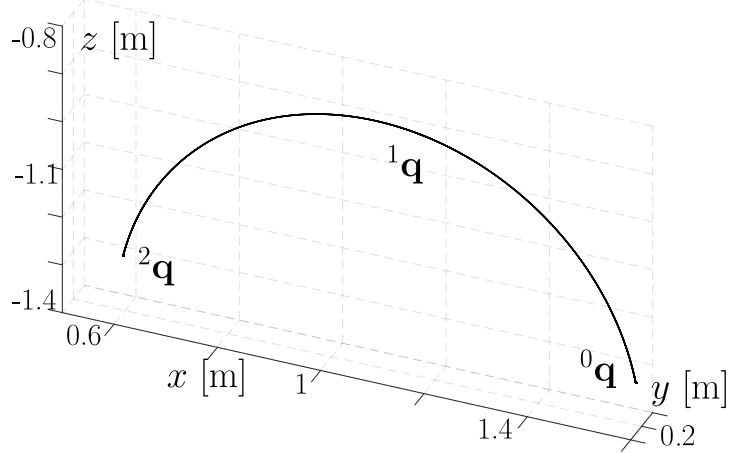

Figure 11. Circular geometric paths.

led to delays in the actual uncoiling process, thus resulting in a slight modification of the cable effective length during experiment. In addition, a steeper slope means that higher frequency responses of the mechanical system can be excited, such as cables axial vibrations, that were not considered in this work. These effects could have had an impact on the limited oscillation that can be observed in the video file attached. The second and third transitions required more limited slope changes for cable lengths in the $R T R$ case compared to STD trajectories, and no detectable oscillations appear in the platform during experiments.

2) Circular Arc Trajectories: In the second example, circular geometric paths connecting the set-points are considered. The $s$-th transition is parametrized by the parametric equation of a circular arc passing through 3 points:

$$
\begin{aligned}
{ }^{s} \mathbf{p}(u)=\mathbf{c}+r_{c} \mathbf{x}_{c} \cos \left[{ }^{s-1} \beta+\left({ }^{s} \beta-{ }^{s-1} \beta\right) u\right]+ \\
+r_{c} \mathbf{y}_{c} \sin \left[{ }^{s-1} \beta+\left({ }^{s} \beta-{ }^{s-1} \beta\right) u\right]
\end{aligned}
$$

where $\mathbf{c}$ is the center of the circle passing through 3 points ${ }^{0} \mathbf{p},{ }^{1} \mathbf{p}$ and ${ }^{2} \mathbf{p}, r_{c}$ is its radius, $\mathbf{z}_{c}$ is a unit vector normal to the circle plane, $\mathbf{x}_{c}=\left({ }^{0} \mathbf{p}-\mathbf{c}\right) /\left\|^{0} \mathbf{p}-\mathbf{c}\right\|$, and $\mathbf{y}_{c}=\mathbf{z}_{c} \times \mathbf{x}_{c}$. In addition, angle ${ }^{s} \beta$ is defined as:

$$
{ }^{s} \beta=\arccos \left[\frac{\left({ }^{s} \mathbf{p}-\mathbf{c}\right) \cdot\left({ }^{0} \mathbf{p}-\mathbf{c}\right)}{r_{c}^{2}}\right] \mathrm{rad}
$$

The solution of the BVP defined by Eq. [50 for $s=1,2,3$ is found starting from an initial guess $\boldsymbol{\kappa}=\mathbf{0}_{6 \times 1}$ in averagely 2 min and the results are summarized in Eq. (64) and Fig. 12 and 13

$$
{ }^{1} \boldsymbol{\kappa}=\left[\begin{array}{c}
-4.403 \\
13.032 \\
-23.118 \\
22.991 \\
-11.721 \\
2.386
\end{array}\right] \quad{ }^{2} \boldsymbol{\kappa}=\left[\begin{array}{c}
-18.691 \\
61.437 \\
-107.706 \\
102.695 \\
-50.163 \\
9.812
\end{array}\right] \quad{ }^{3} \boldsymbol{\kappa}=\left[\begin{array}{c}
-3.924 \\
8.662 \\
-10.559 \\
7.231 \\
-2.606 \\
0.384
\end{array}\right]
$$

It can be noted in the planning results that in the first transition the slope of the cable commanded lengths is less steep for RTR trajectories than for STD ones, and the transition occurs smoothly without any oscillation left. The contrary occurs in the second and the third transition. In the second one (Figs. 12a to 12c, this fact results in a very quick final movement that excites axial vibrations in cables, which are rapidly damped out, but cause a limited oscillatory behavior (this phenomenon can be clearly observed in the attached video file at 1min40s). Still, the amplitude of these oscillations is not detected by the feedback measurement system, whereas the wide oscillations in the STD case are apparent. The third and last transitions, in the STD case, display one dangerous effect: the possibility of an unstable behavior of the $e$-e due to the lack of constraint (namely, the unactuated DoFs evolve with no predetermined boundaries). While transitioning, the platform uncontrolled orientation (see Fig. 14) was rapidly changing, almost leading the platform to tip over. In the RTR case, instead, the platform slightly oscillates during the transition, and arrives at the rest position with a very limited residual oscillation (Figs. $15 \mathrm{a}$ and $15 \mathrm{~b}$ show 


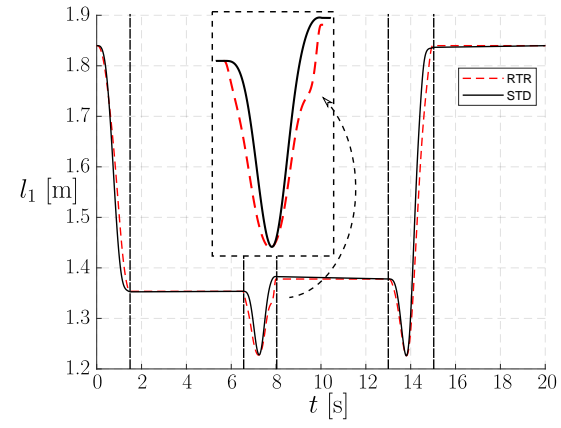

(a) First cable

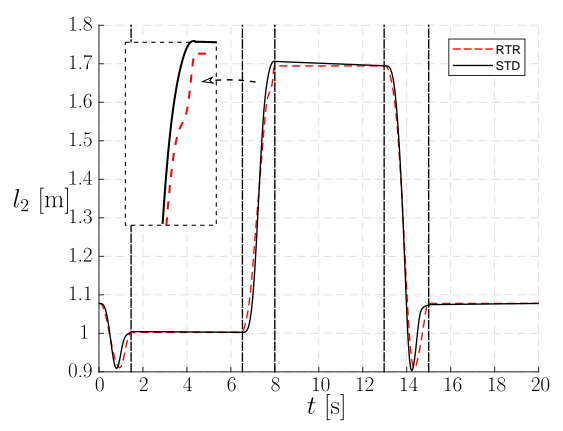

(b) Second cable

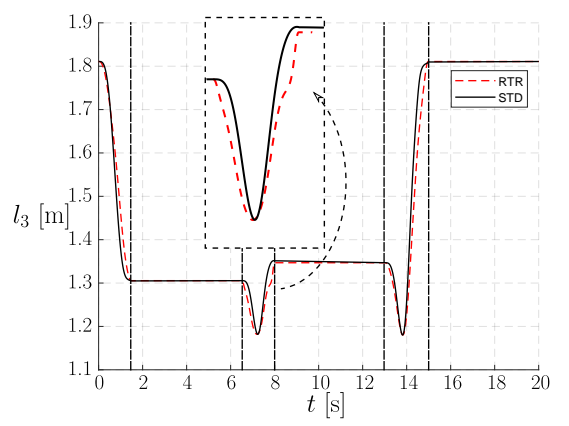

(c) Third cable

Figure 12. Actuator position set-points for RTR and STD trajectories, in the case of circular paths. Transitions between set-points are delimited by vertical dashed lines

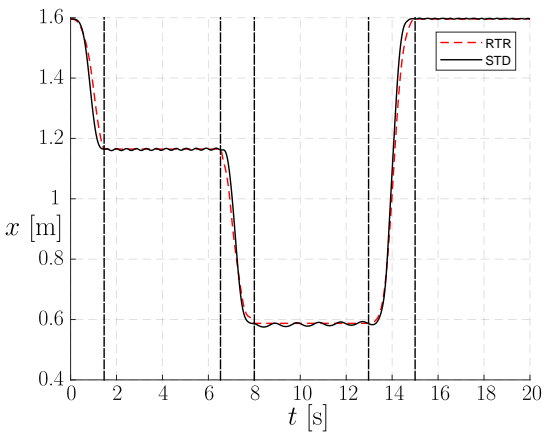

(a) $x$ coordinate

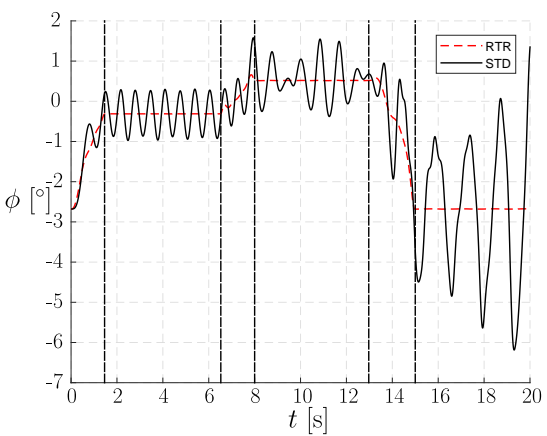

(d) $\phi$ Euler angle

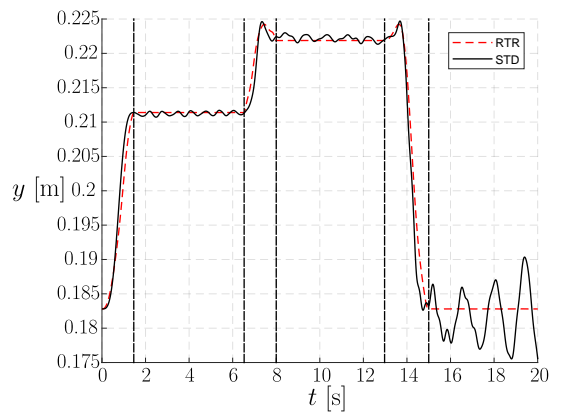

(b) $y$ coordinate

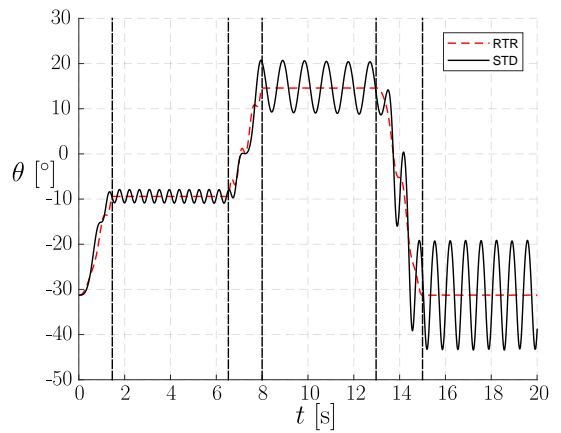

(e) $\theta$ Euler angle

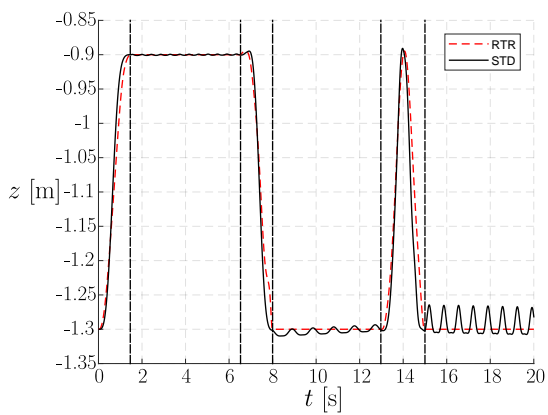

(c) $z$ coordinate

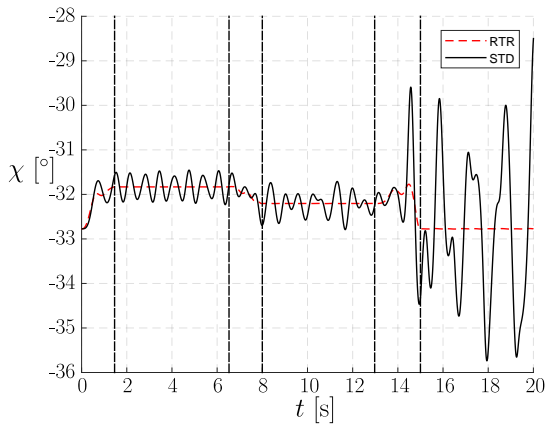

(f) $\chi$ Euler angle

Figure 13. Computed end-effector pose for RTR and STD trajectories, in the case of circular paths. Transitions between set-points are delimited by vertical dashed lines

an enhanced view of the most critical oscillations observed during experiments, for $8 \mathrm{~s}<t<13 \mathrm{~s}$ ).

It is clear from the results presented in this section that the $e$ - $e$ may be brought to rest after a transition from one set-point to another only by an accurate trajectory planning that takes into account the internal dynamics of the system.

\section{CONCLUSION}

This paper presented the trajectory planning for underactuated CDPRs in the case of rest-to-rest motions when the motion time and the path geometry are prescribed. If an arbitrary motion is prescribed for a suitable subset of the end-effector coordinates, the constraint deficiency on the end-effector motions leads to the impossibility of bringing the system at rest in a prescribed time. In addition, an unbounded oscillatory motion of the end-effector may arise. After formulating the problem as a boundary value problem $(B V P)$ with free parameters, a solution technique was proposed and implemented for offline planning. The actuated cable lengths resulting from planning were used as position set-points for the actuators of a $C D P R$ prototype in experiments. Numerical and video results, showing different implications of the proposed technique with respect to a standard planning, were provided and discussed. Experimental results are satisfactory, even without a closed-loop control on the platform pose in the Cartesian space, and have indicated possible ways to improve such planning methodology, from both a modeling and a practical implementation point of view. In the future, elastic effects and explicit limits on cable tensions and accelerations (resulting from safety requirements and motor dynamics) will be included in the planning algorithm, since they 


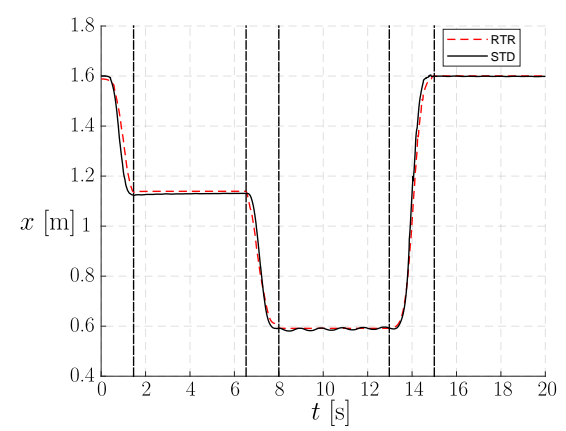

(a) $x$ coordinate

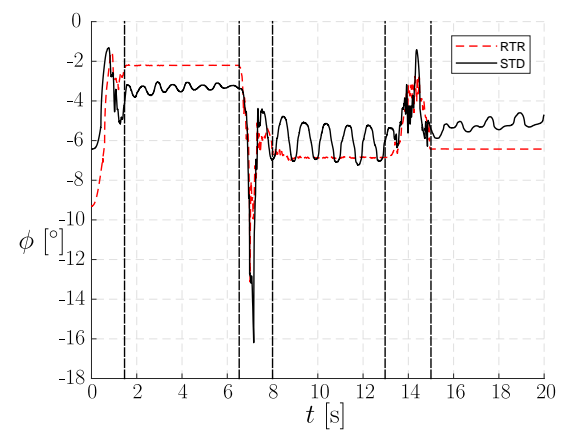

(d) $\phi$ Euler angle

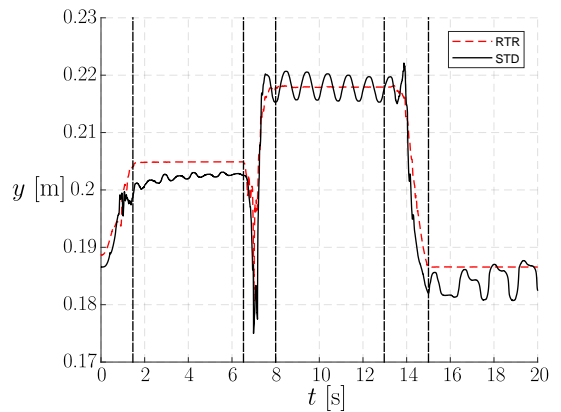

(b) $y$ coordinate

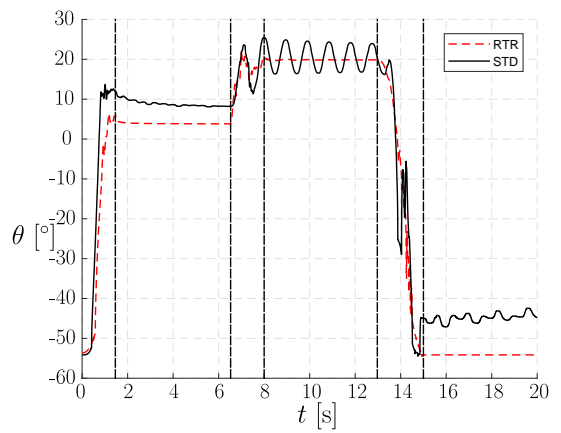

(e) $\theta$ Euler angle

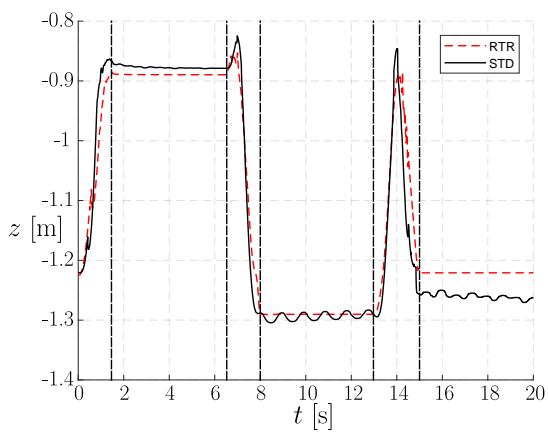

(c) $z$ coordinate

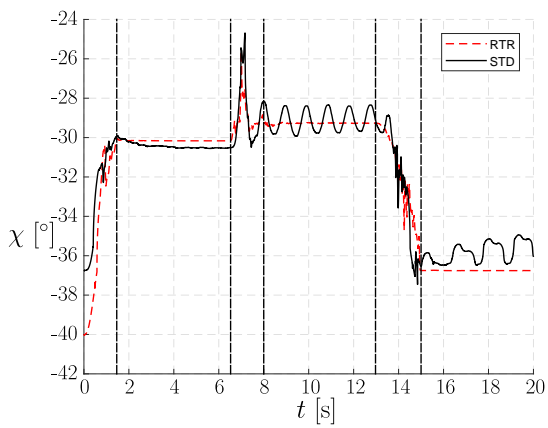

(f) $\chi$ Euler angle

Figure 14. Measured end-effector pose for RTR and STD trajectories, in the case of circular paths. Transitions between set-points are delimited by vertical dashed lines

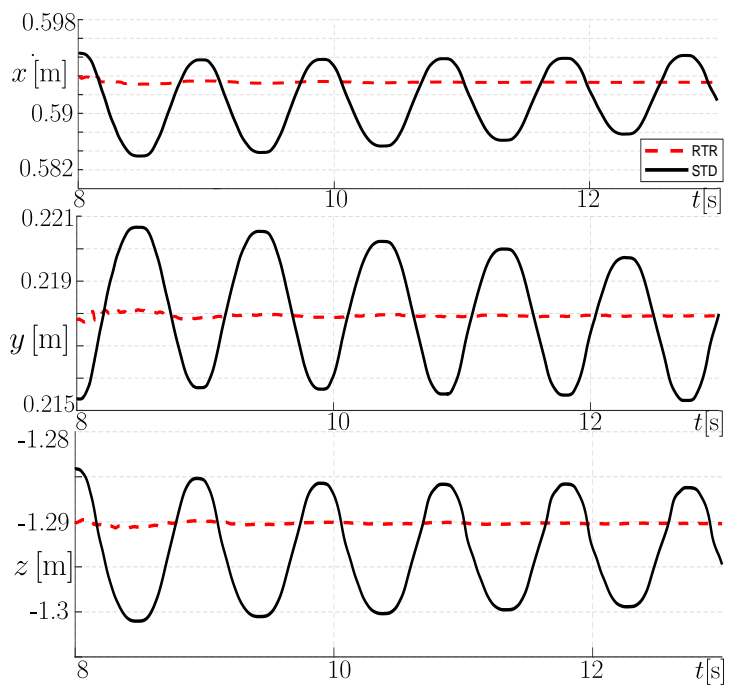

(a) End-effector reference position

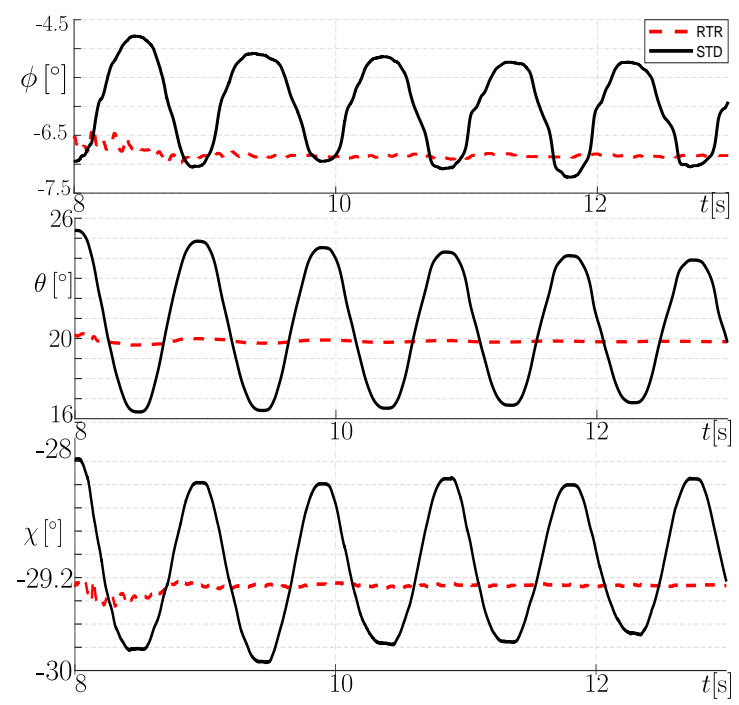

(b) End-effector Euler angles

Figure 15. Enhanced view of most critical oscillations during circular paths.

can practically limit the implementation of the method proposed in this paper. In addition, some components of our prototypes will be engineered so as to provide better adherence to the mathematical model used for planning. In the end, the possibility of a closed-loop feedback control of the platform pose will be addressed in order to provide a comprehensive solution for the dynamic operation of underactuated CDPRs.

\section{APPENDIX}

\section{END-EFFECTOR DYNAMIC EQUATIONS}

Consider Eqs. (32) and (33), which are reproposed hereafter for the sake of convenience:

$$
\begin{array}{r}
m \ddot{\mathbf{r}}-\mathbf{f}_{G}-\mathbf{f}_{C}=\mathbf{0} \\
\mathbf{I}_{G} \boldsymbol{\alpha}+\boldsymbol{\omega} \times\left(\mathbf{I}_{G} \boldsymbol{\omega}\right)+m \mathbf{r}^{\prime} \times \ddot{\mathbf{r}}-\left(\mathbf{m}_{G}+\mathbf{r}^{\prime} \times \mathbf{f}_{G}\right)-\mathbf{m}_{C}=\mathbf{0}
\end{array}
$$

Substituting the expression of $\ddot{\mathbf{r}}$ given in Eq. [19] in Eqs. 
65) and 66 yields, respectively:

$$
\begin{array}{r}
m \ddot{\mathbf{p}}-m \mathbf{r}^{\prime} \times \boldsymbol{\alpha}-m \boldsymbol{\omega} \times\left(\mathbf{r}^{\prime} \times \boldsymbol{\omega}\right)-\mathbf{f}_{G}-\mathbf{f}_{C}=\mathbf{0} \\
\mathbf{I}_{G} \boldsymbol{\alpha}-m \mathbf{r}^{\prime} \times\left(\mathbf{r}^{\prime} \times \boldsymbol{\alpha}\right)+m \mathbf{r}^{\prime} \times \ddot{\mathbf{p}}+\boldsymbol{\omega} \times\left(\mathbf{I}_{G} \boldsymbol{\omega}\right)+ \\
-m \mathbf{r}^{\prime} \times\left[\boldsymbol{\omega} \times\left(\mathbf{r}^{\prime} \times \boldsymbol{\omega}\right)\right]-\left(\mathbf{m}_{G}+\mathbf{r}^{\prime} \times \mathbf{f}_{G}\right)-\mathbf{m}_{C}=\mathbf{0}
\end{array}
$$

Since:

$$
\mathbf{r}^{\prime} \times\left[\omega \times\left(\mathbf{r}^{\prime} \times \omega\right)\right]=\omega \times\left[\mathbf{r}^{\prime} \times\left(\mathbf{r}^{\prime} \times \omega\right)\right]
$$

Eq. 68 can be also written as:

$$
\begin{aligned}
& \mathbf{I}_{G} \boldsymbol{\alpha}-m \mathbf{r}^{\prime} \times\left(\mathbf{r}^{\prime} \times \boldsymbol{\alpha}\right)+m \mathbf{r}^{\prime} \times \ddot{\mathbf{p}}+ \\
& +\boldsymbol{\omega} \times\left[\mathbf{I}_{G} \boldsymbol{\omega}-m \mathbf{r}^{\prime} \times\left(\mathbf{r}^{\prime} \times \boldsymbol{\omega}\right)\right]-\left(\mathbf{m}_{G}+\mathbf{r}^{\prime} \times \mathbf{f}_{G}\right)-\mathbf{m}_{C}=\mathbf{0}
\end{aligned}
$$

By introducing:

$$
\mathbf{I}_{P}=\mathbf{I}_{G}-m \tilde{\mathbf{r}}^{\prime} \tilde{\mathbf{r}}^{\prime}
$$

and recalling Eqs. 21) and (22), Eqs. (67) and (70) can be rewritten in matrix form as:

$$
\begin{aligned}
m \ddot{\mathbf{p}}-m \tilde{\mathbf{r}}^{\prime} \mathbf{H} \ddot{\boldsymbol{\epsilon}}-m\left(\tilde{\mathbf{r}}^{\prime} \dot{\mathbf{H}}+\tilde{\boldsymbol{\omega}} \tilde{\mathbf{r}}^{\prime} \mathbf{H}\right) \dot{\boldsymbol{\epsilon}}-\mathbf{f}_{G}-\mathbf{f}_{C}=\mathbf{0} \\
-m \tilde{\mathbf{r}}^{\prime T} \ddot{\mathbf{p}}+\mathbf{I}_{P} \mathbf{H} \ddot{\boldsymbol{\epsilon}}+\left(\mathbf{I}_{P} \dot{\mathbf{H}}+\tilde{\boldsymbol{\omega}} \mathbf{I}_{P} \mathbf{H}\right) \dot{\boldsymbol{\epsilon}} \\
-\mathbf{m}_{G}-\tilde{\mathbf{r}}^{\prime} \mathbf{f}_{G}-\mathbf{m}_{C}=\mathbf{0}
\end{aligned}
$$

namely (cf. Eq. [34):

$$
\mathbf{M}^{\prime} \ddot{\mathbf{q}}-\mathbf{s}^{\prime}+\mathbf{J}_{v}^{T} \boldsymbol{\tau}=\mathbf{0}
$$

where:

$$
\begin{gathered}
\mathbf{M}^{\prime}=\left[\begin{array}{cc}
m \mathbf{I}_{3} & -m \tilde{\mathbf{r}}^{\prime} \mathbf{H} \\
-m \tilde{\mathbf{r}}^{\prime T} & \mathbf{I}_{P} \mathbf{H}
\end{array}\right] \\
\mathbf{s}^{\prime}=\left[\begin{array}{c}
m\left(\tilde{\mathbf{r}}^{\prime} \dot{\mathbf{H}}+\tilde{\boldsymbol{\omega}} \tilde{\mathbf{r}}^{\prime} \mathbf{H}\right) \dot{\boldsymbol{\epsilon}}+\mathbf{f}_{G} \\
-\left(\mathbf{I}_{P} \dot{\mathbf{H}}+\tilde{\boldsymbol{\omega}} \mathbf{I}_{P} \mathbf{H}\right) \dot{\boldsymbol{\epsilon}}+\mathbf{m}_{G}+\tilde{\mathbf{r}}_{G}^{\prime} \mathbf{f}_{G}
\end{array}\right]
\end{gathered}
$$

However, matrix $\mathbf{M}^{\prime}$ is not symmetric, and this could lead to numerical difficulties. We can overcome this obstacle by pre-multiplying Eq. 74] by:

$$
\mathbf{H}^{\prime}=\left[\begin{array}{cc}
\mathbf{I}_{3} & \mathbf{0}_{3} \\
\mathbf{0}_{3} & \mathbf{H}^{T}
\end{array}\right]
$$

where $\mathbf{0}_{3}$ is the $3 \times 3$ zero matrix, finally obtaining:

$$
\mathbf{M} \ddot{\mathbf{q}}-\mathbf{s}-\mathbf{B} \boldsymbol{\tau}=\mathbf{0}
$$

where:

$$
\mathbf{M}=\mathbf{H}^{\prime} \mathbf{M}^{\prime}, \quad \mathbf{s}=\mathbf{H}^{\prime} \mathbf{s}^{\prime}, \quad \mathbf{B}=-\mathbf{H}^{\prime} \mathbf{J}_{v}^{T}=-\mathbf{J}_{q}^{T}
$$

\section{ACKNOWLEDGMENT}

The authors thank Dr. Giovanni Mottola for his help in shooting and editing the video attached to this paper.

\section{REFERENCES}

[1] E. Idá, A. Berti, T. Bruckmann, and M. Carricato, "Rest-to-Rest trajectory planning for planar underactuated cable-driven parallel robots," in Cable-Driven Parallel Robots, C. Gosselin, P. Cardou, T. Bruckmann, and A. Pott, Eds. Cham: Springer, 2018, pp. 207-218.

[2] M. Conconi and M. Carricato, "A new assessment of singularities of parallel kinematic chains," IEEE Transactions on Robotics, vol. 25 no. 4, pp. 757-770, Aug 2009.

[3] M. Carricato and J. P. Merlet, "Stability analysis of underconstrained cable-driven parallel robots," IEEE Transactions on Robotics, vol. 29, no. 1, pp. 288-296, Feb 2013.

[4] M. Yamamoto, N. Yanai, and A. Mohri, "Trajectory control of incompletely restrained parallel-wire-suspended mechanism based on inverse dynamics," IEEE Transactions on Robotics, vol. 20, no. 5, pp. 840-850, Oct 2004.

[5] T. Heyden and C. Woernle, "Dynamics and flatness-based control of a kinematically undetermined cable suspension manipulator," Multibody System Dynamics, vol. 16, no. 2, p. 155, Aug 2006.

[6] A. Pott, "An algorithm for real-time forward kinematics of cabledriven parallel robots," in Advances in Robot Kinematics: Motion in Man and Machine, J. Lenarcic and M. M. Stanisic, Eds. Dordrecht: Springer, 2010, pp. 529-538.

[7] _ "Influence of pulley kinematics on cable-driven parallel robots," in Latest Advances in Robot Kinematics, J. Lenarcic and M. Husty, Eds. Dordrecht: Springer, 2012, pp. 197-204.

[8] J. Pusey, A. Fattah, S. Agrawal, and E. Messina, "Design and workspace analysis of a 6-6 cable-suspended parallel robot," Mechanism and Machine Theory, vol. 39, no. 7, pp. 761 - 778, 2004.

[9] P. Bosscher, A. T. Riechel, and I. Ebert-Uphoff, "Wrench-feasible workspace generation for cable-driven robots," IEEE Transactions on Robotics, vol. 22, no. 5, pp. 890-902, Oct 2006.

[10] G. Barrette and C. M. Gosselin, "Determination of the dynamic workspace of cable-driven planar parallel mechanisms," Journal of Mechanical Design, vol. 127, no. 2, pp. 242-248, 2005.

[11] S. Bouchard, C. Gosselin, and B. Moore, "On the ability of a cabledriven robot to generate a prescribed set of wrenches," Journal of Mechanisms and Robotics, vol. 2, no. 1, p. 011010, 2010.

[12] M. Gouttefarde, D. Daney, and J. Merlet, "Interval-analysis-based determination of the wrench-feasible workspace of parallel cabledriven robots," IEEE Transactions on Robotics, vol. 27, no. 1, pp. 1-13, Feb 2011.

[13] M. Gouttefarde, J. Lamaury, C. Reichert, and T. Bruckmann, "A versatile tension distribution algorithm for $n$-dof parallel robots driven by $n+2$ cables," IEEE Transactions on Robotics, vol. 31, no. 6, pp. 1444 1457, Dec 2015

[14] C. Gosselin and S. Foucault, "Dynamic point-to-point trajectory planning of a two-dof cable-suspended parallel robot," IEEE Transactions on Robotics, vol. 30, no. 3, pp. 728-736, June 2014.

[15] G. Mottola, C. Gosselin, and M. Carricato, "Dynamically feasible periodic trajectories for generic spatial three-degree-of-freedom cablesuspended parallel robots," Journal of Mechanisms and Robotics, vol. 10 , no. 3,2018

[16] X. Jiang, E. Barnett, and C. Gosselin, "Dynamic point-to-point trajectory planning beyond the static workspace for six-dof cablesuspended parallel robots," IEEE Transactions on Robotics, vol. 34 no. 3, pp. 781-793, 2018.

[17] N. Zhang, W. Shang, and S. Cong, "Dynamic trajectory planning for a spatial 3-dof cable-suspended parallel robot," Mechanism and Machine Theory, vol. 122, pp. 177 - 196, 2018.

[18] P. Dion-Gauvin and C. Gosselin, "Dynamic point-to-point trajectory planning of a three-dof cable-suspended mechanism using the hypocycloid curve," IEEE/ASME Transactions on Mechatronics, vol. 23 no. 4, pp. 1964-1972, 2018.

[19] G. Mottola, C. Gosselin, and M. Carricato, "Dynamically feasible motions of a class of purely-translational cable-suspended paralle robots," Mechanism and Machine Theory, vol. 132, pp. 193-206, 2019.

[20] J.-P. Merlet, "Some properties of the Irvine cable model and their use for the kinematic analysis of cable-driven parallel robots," Mechanism and Machine Theory, vol. 135, pp. 271 - 280, 2019.

[21] S. Perreault, P. Cardou, C. M. Gosselin, and M. J.-D. Otis, "Geometric determination of the interference-free constant-orientation workspace of parallel cable-driven mechanisms," Journal of Mechanisms and Robotics, vol. 2, no. 3, p. 031016, 2010.

[22] J. Fink, N. Michael, S. Kim, and V. Kumar, "Planning and control for cooperative manipulation and transportation with aerial robots," The 
International Journal of Robotics Research, vol. 30, no. 3, pp. 324-334, 2011.

[23] L. Barbazza, D. Zanotto, G. Rosati, and S. K. Agrawal, "Design and optimal control of an underactuated cable-driven micro-macro robot," IEEE Robotics and Automation Letters, vol. 2, no. 2, pp. 896903, April 2017.

[24] G. Abbasnejad and M. Carricato, "Direct geometrico-static problem of underconstrained cable-driven parallel robots with $n$ cables," IEEE Transactions on Robotics, vol. 31, no. 2, pp. 468-478, April 2015.

[25] A. Berti, J.-P. Merlet, and M. Carricato, "Solving the direct geometricostatic problem of underconstrained cable-driven parallel robots by interval analysis," The International Journal of Robotics Research vol. 35, no. 6, pp. 723-739, 2016.

[26] M. Fliess, J. Lévine, P. Martin, and P. Rouchon, "Flatness and defect of non-linear systems: introductory theory and examples," International Journal of Control, vol. 61, no. 6, pp. 1327-1361, 1995.

[27] D. Cunningham and H. H. Asada, "The winch-bot: A cablesuspended, under-actuated robot utilizing parametric self-excitation," in 2009 IEEE International Conference on Robotics and Automation, May 2009, pp. 1844-1850.

[28] N. Zoso and C. Gosselin, "Point-to-point motion planning of a parallel 3-dof underactuated cable-suspended robot," in 2012 IEEE International Conference on Robotics and Automation, May 2012, pp. 2325-2330.

[29] J. Park, O. Kwon, and J. H. Park, "Anti-sway trajectory generation of incompletely restrained wire-suspended system," Journal of Mechanical Science and Technology, vol. 27, no. 10, pp. 3171-3176, Oct 2013.

[30] S. W. Hwang, J.-H. Bak, J. Yoon, J. H. Park, and J.-O. Park, "Trajectory generation to suppress oscillations in under-constrained cabledriven parallel robots," Journal of Mechanical Science and Technology, vol. 30, no. 12, pp. 5689-5697, Dec 2016.

[31] D. Surdilovic, J. Radojicic, and J. Krüger, "Geometric stiffness analysis of wire robots: A mechanical approach," in Cable-Driven Parallel Robots, T. Bruckmann and A. Pott, Eds. Berlin, Heidelberg: Springer, 2013, pp. 389-404.

[32] A. Pott, Cable-driven parallel robots: theory and application. Springer, 2018, vol. 120.

[33] G. Oriolo and Y. Nakamura, "Control of mechanical systems with second-order nonholonomic constraints: underactuated manipulators," in 30th IEEE Conference on Decision and Control, Dec 1991, pp. 2398-2403.

[34] A. Isidori, Nonlinear control systems. Springer, 2013.

[35] R. Seifried, Dynamics of underactuated multibody systems: modeling, control and optimal design. Springer, 2013.

[36] K. Graichen, V. Hagenmeyer, and M. Zeitz, "A new approach to inversion-based feedforward control design for nonlinear systems," Automatica, vol. 41, no. 12, pp. 2033 - 2041, 2005.

[37] K. Graichen and M. Zeitz, "Feedforward control design for finitetime transition problems of non-linear mimo systems under input constraints," International Journal of Control, vol. 81, no. 3, pp. 417427, 2008.

[38] J. S. Short, A. N. Poo, C. Y. Lai, P. Y. Tao, and M. H. Ang, "A generalized underactuated robot system inversion method using hamiltonian formalism," in 2015 IEEE International Conference on Advanced Intelligent Mechatronics (AIM), July 2015, pp. 1415-1420.

[39] A. Piazzi and A. Visioli, "Optimal noncausal set-point regulation of scalar systems," Automatica, vol. 37, no. 1, pp. 121 - 127, 2001.

[40] J. Bobrow, S. Dubowsky, and J. Gibson, "Time-optimal control of robotic manipulators along specified paths," The International Journal of Robotics Research, vol. 4, no. 3, pp. 3-17, 1985.

[41] L. F. Shampine, J. Kierzenka, and M. W. Reichelt, "Solving boundary value problems for ordinary differential equations in Matlab with bvp4c," Tutorial notes, vol. 2000, pp. 1-27, 2000.

[42] U. M. Ascher and L. R. Petzold, Computer methods for ordinary differential equations and differential-algebraic equations. Siam, 1998.

[43] J.-P. Merlet, "Direct kinematics of cdpr with extra cable orientation sensors: The 2 and 3 cables case with perfect measurement and ideal or elastic cables," in Cable-Driven Parallel Robots, C. Gosselin, P. Cardou, T. Bruckmann, and A. Pott, Eds. Cham: Springer, 2018, pp. 180-191.

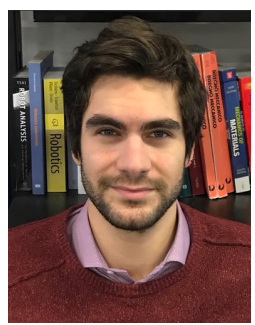

Edoardo Idà received the M.Sc. degree (with honors) in Mechanical Engineering in 2017 from the University of Bologna, and is currently a Ph.D. student in Mechanics and Advanced Engineering Sciences at the same university. His research interests focus on cable-driven robotic systems.

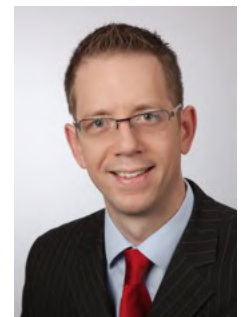

Tobias Bruckmann received the Dipl.-Ing. in Mechanical Engineering from the University of Duisburg-Essen, Duisburg, Germany, in 2004 and the Dr.-Ing. In 2010. He is currently working as a Lecturer and Research Group Leader at the Chair of Mechatronics, University of DuisburgEssen, Germany, with a focus on robotics, including cable-driven parallel manipulators. Dr. Bruckmann is co-initiator and member of the scientific committee of the "Int. Conf. on CableDriven Parallel Robots". Since 2015 he is member of the scientific committees of the IFToMM D-A-CH Conferences and, since 2017, a member of the scientific committee of the IFToMM Int Symposium on Robotics \& Mechatronics. Dr. Bruckmann is member of VDI/VDI-GMA Technical Committees 4.15 "Mechatronik" and 4.10 "Interdisziplinäre Produktentstehung" and the IFToMM Technical Committee for Robotics and Mechatronics. He edited three books and co-authored over 60 scientific articles and conference contributions.

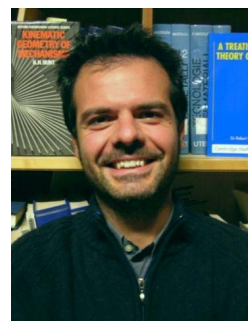

Marco Carricato received the M.Sc. degree (with honors) in Mechanical Engineering in 1998 and the Ph.D. degree in mechanics of machines in 2002. He has been with the University of Bologna since 2004. He is currently an associate professor. He was visiting researcher at the University of Florida (USA), at Laval University (Canada), at the University of Guanajuato (Mexico), at INRIASophia Antipolis (France), at the Ecole Centrale of Nantes (France), and at the Hong Kong University of Science and Technology. He is an associate editor of the journal Mechanism and Machine Theory. He was awarded the AIMETA Junior Prize 2011 by the Italian Association of Theoretical and Applied Mechanics for outstanding results in the field of mechanics of machines. His research interests include robotic systems, servo-actuated automatic machinery and the theory of mechanisms. 